\title{
A One-Dimensional Flow Analysis for the Prediction of Centrifugal Pump Performance Characteristics
}

\author{
Mohammed Ahmed El-Naggar \\ Department of Mechanical Power Engineering, Mansoura University, Mansoura 35516, Egypt \\ Correspondence should be addressed to Mohammed Ahmed El-Naggar; naggarm@mans.edu.eg
}

Received 25 June 2013; Accepted 29 August 2013

Academic Editor: Shigenao Maruyama

Copyright (c) 2013 Mohammed Ahmed El-Naggar. This is an open access article distributed under the Creative Commons Attribution License, which permits unrestricted use, distribution, and reproduction in any medium, provided the original work is properly cited.

\begin{abstract}
A one-dimensional flow procedure for analytical study of centrifugal pump performance is done applying the principle theories of turbomachines. Euler equation and energy equation are manipulated to find pump performance parameters at different discharge coefficients. Fluid slippage loss at impeller exit and volute loss are estimated. The fluid slippage is modeled by the slip factor approach using Wiesner empirical expression. The volute loss model counts friction loss associated with the volute throw flow velocity, diffusion friction loss due to circulation associated with volute flow, loss due to vanishing of radial flow at volute outlet, and loss inside pump volute throat. Models for impeller hydraulic friction power loss, disk friction power loss, internal flow leakage power loss, and inlet shock circulation power loss are considered by suitable models. Pump internal volumetric flow leakage and volumetric efficiency are related to pump geometry and flow properties. The procedure adopted in this paper is capable of obtaining performance characteristic curves of centrifugal pump in a dimensionless form. Pump head coefficient, manometric efficiency, power coefficient, and required NPSH are characterized. The predicted coefficients and obtained performance curves are consistent with experimental characteristics of centrifugal pump.
\end{abstract}

\section{Introduction}

Centrifugal pumps are used in various applications and are integral to many industries. Yet, in spite of their prevalence and relatively simple configurations compared to other turbomachines, designing an efficient and durable pump remains a challenge.

The design of centrifugal pumps is still determined empirically because it relies on the use of a number of experimental and statistical rules. However, during the last few years, the design and performance analysis of turbomachinery have experienced great progress due to the joint evolution of computer power and the accuracy of numerical methods.

The one-dimensional performance analysis has proved to be an effective and important approach on pump design [1]. Analytical calculations of pump characteristics depend on geometrical dimensions of pump and losses models in different parts of pump. A series of formulae for calculating losses exist [2-5], but they lack accuracy when applied to centrifugal pumps.
In this work, suggested models for calculating several losses in pump are introduced to examine its validity in evaluating pump performance. This paper is an effort towards theoretically obtaining accurate centrifugal pump performance characteristics. Pump characteristics and parameters are presented in dimensionless forms. It presents a onedimensional flow analysis procedure towards obtaining optimum centrifugal pump design parameters.

\section{Theoretical Analysis}

The pump flow coefficient $\psi$ and pump speed coefficient $\varphi$ are defined as

$$
\psi=\frac{V_{r_{2}}}{\sqrt{2 g H}}, \quad \varphi=\frac{u_{2}}{\sqrt{2 g H}},
$$

where $H$ is the pump manometric head, $V_{r_{2}}$ is the flow radial velocity at impeller outlet, and $u_{2}$ is the tangential velocity at outlet of impeller. 

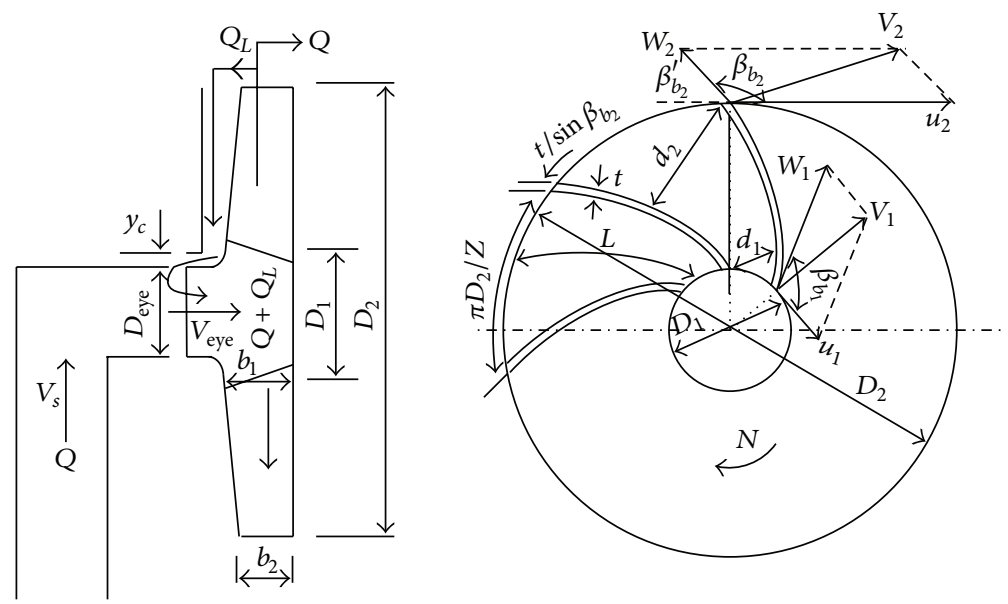

Figure 1: Pump impeller notations.

2.1. Pump Leakage. Due to the difference between the outlet pressure and the inlet pressure of the impeller, a portion of impeller outlet flow rate, $Q_{L}$, returns to the impeller inlet from the existing clearances between the impeller and the casing, Figure 1. This internal discharge leakage, $Q_{L}$, causes some losses as the flow rate through the impeller $\left(Q+Q_{L}\right)$ is greater than the pump useful outlet discharge $Q$.

The volumetric efficiency $\eta_{\mathrm{vol}}$ of pump is defined as the ratio of pump outlet discharge to the impeller discharge:

$$
\eta_{\mathrm{vol}}=\frac{Q}{Q_{i}}=\frac{Q}{Q+Q_{L}} \quad \text { or } \quad \frac{Q_{L}}{Q}=\frac{1}{\eta_{\text {vol }}}-1,
$$

in which

$$
Q_{i}=Q+Q_{L}=V_{r_{2}} \cdot \pi D_{2} b_{2} \varepsilon_{2}=V_{r_{1}} \cdot \pi D_{1} b_{1} \varepsilon_{1},
$$

where $b_{1}$ is the blade width at inlet, $D_{1}$ is the impeller diameter at inlet, $\varepsilon_{1}$ is the blade thickness coefficient at impeller inlet, $\varepsilon_{1}=1-(Z / \pi)\left(\left(t / \sin \beta_{b_{1}}\right) / D_{1}\right), b_{2}$ is the blade width at outlet, $D_{2}$ is the impeller diameter at outlet, $\varepsilon_{2}$ is the blade thickness coefficient at impeller outlet, $\varepsilon_{2}=1-(Z / \pi)\left(\left(t / \sin \beta_{b_{2}}\right) / D_{2}\right), t$ is the blade thickness, $Z$ is the number of blades, $\beta_{b_{2}}$ is the blade angle at impeller outlet, and $\beta_{b_{1}}$ is the blade angle at impeller inlet. The value of $\varepsilon_{2}$ is about 0.95 [6]. The relationship between $\varepsilon_{1}$ and $\varepsilon_{2}$ with constant blade thickness is given as

$$
\varepsilon_{1}=1-\frac{\left(1-\varepsilon_{2}\right)}{\left(D_{1} / D_{2}\right)\left(\sin \beta_{b_{1}} / \sin \beta_{b_{2}}\right)} .
$$

The impeller inlet flow velocity coefficient $C_{V_{1}}=$ $V_{1} / \sqrt{2 g H}$ is calculated from (3) dividing both sides by $\sqrt{2 g H}$ and assuming that the flow enters the impeller without swirl $\left(V_{1}=V_{r_{1}}\right)$ :

$$
C_{V_{1}}=\frac{\psi}{\left(D_{1} / D_{2}\right)\left(b_{1} / b_{2}\right)\left(\varepsilon_{1} / \varepsilon_{2}\right)}
$$

The pump discharge coefficient is $C_{Q}=Q /\left((N / 60) D_{2}^{3}\right)$, and substituting for $Q$ from (2) and (3);

$$
C_{Q}=\eta_{\mathrm{vol}} \varepsilon_{2} \pi^{2} \frac{b_{2}}{D_{2}} \cdot \frac{\psi}{\varphi} .
$$

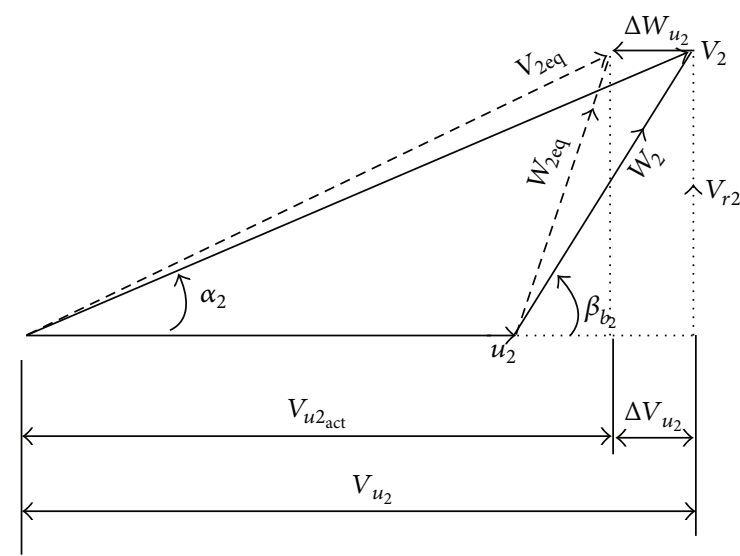

FIGURE 2: Velocity diagram at impeller outlet.

2.2. Outlet Velocity Diagram. A fluid slippage occurs at impeller exit due to the relative rotation of fluid in a direction opposite to that of impeller. A slip factor $\sigma$ defined as $\sigma=$ $V_{u_{2} \text { act }} / V_{u_{2}}$ could be estimated later in Section 2.3.

From the velocity diagram at the impeller outlet, Figure 2, the tangential component of the outlet flow absolute velocity is given as

$$
V_{u_{2}}=V_{r_{2}} \cot \alpha_{2}=u_{2}+V_{r_{2}} \cot \beta_{b_{2}} .
$$

The ratio of outlet swirl velocity to outlet tangential velocity is

$$
\frac{V_{u_{2}}}{u_{2}}=1+\frac{V_{r_{2}}}{u_{2}} \cot \beta_{b_{2}}=1+\frac{\psi}{\varphi} \cot \beta_{b_{2}},
$$

and thus,

$$
\frac{V_{u_{\mathrm{act}}}}{u_{2}}=\frac{\sigma \cdot V_{u_{2}}}{u_{2}}=\sigma\left(1+\frac{\psi}{\varphi} \cot \beta_{b_{2}}\right) .
$$

The outlet tangential velocity (from (7)) and the pump speed coefficient $\varphi=u_{2} / \sqrt{2 g H}$ are given as

$$
\begin{aligned}
u_{2} & =V_{r_{2}}\left(\cot \alpha_{2}-\cot \beta_{b_{2}}\right), \\
\varphi & =\psi\left(\cot \alpha_{2}-\cot \beta_{b_{2}}\right) .
\end{aligned}
$$




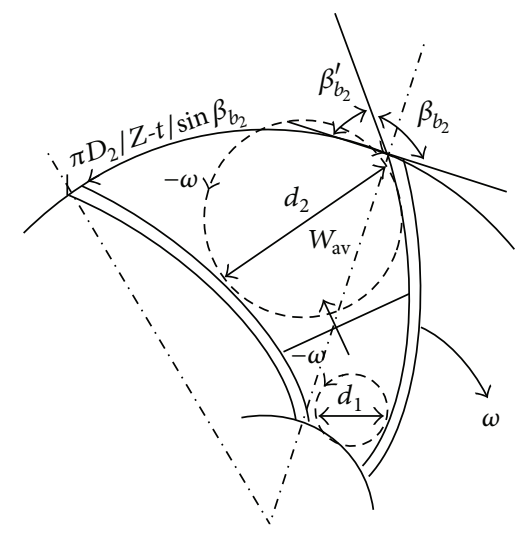

FIgURE 3: Flow model for Stodola slip factor.

From the outlet velocity diagram (Figure 2) and (7), the outlet velocity and the outlet velocity coefficient defined as $C_{V_{2}}=$ $V_{2} / \sqrt{2 g H}$ are

$$
\begin{gathered}
V_{2}^{2}=V_{r_{2}}^{2}+V_{u_{2}}^{2}=V_{r_{2}}^{2}+\left(u_{2}+V_{r_{2}} \cot \beta_{b_{2}}\right)^{2} \\
C_{V_{2}}^{2}=\psi^{2}+\left(\varphi+\psi \cot \beta_{b_{2}}\right)^{2}
\end{gathered}
$$

\subsection{Slip Factor}

2.3.1. The Relative Eddy (Eddy Circulation). A simple explanation for the slip effect in an impeller is obtained from the idea of a relative eddy. Suppose that an irrotational and frictionless fluid flow is possible which passes through an impeller. If the absolute flow enters the impeller without spin, then at outlet the spin of the absolute flow must still be zero. The impeller itself has an angular velocity $\omega$ so that, relative to the impeller, the fluid has an angular velocity of $-\omega$; this is termed the relative eddy. At outlet of impeller, the relative flow, $W_{2}$, can be regarded as a through flow on which a relative eddy is superimposed. The net effect of these two motions is that the average relative flow emerging from the impeller passages is at an angle to the vanes and in a direction opposite to the blade motion.

One of the earliest and simplest expressions for the slip factor was obtained by Stodola, cited in [7]. Referring to Figure 3, the slip velocity due to relative eddy, $\Delta W_{u_{2}}=\Delta V_{u_{2}}=$ $V_{u_{2}}-V_{u_{2} \text { act }}$, is considered to be the product of the relative eddy and the radius $\left(d_{2} / 2\right)$ of a circle which can be inscribed within the channel.

Thus,

$$
\Delta W_{u_{2}}=\omega \frac{d_{2}}{2} .
$$

An approximate expression for $d_{2}$ can be written if the number of blades $Z$ is not small:

$$
\begin{gathered}
d_{2}=\frac{\pi D_{2}}{Z} \sin \beta_{b_{2}}^{\prime}-t=\varepsilon_{2} \frac{\pi D_{2}}{Z} \sin \beta_{b_{2}}, \\
\frac{d_{2}}{D_{2}}=\varepsilon_{2} \frac{\pi}{Z} \sin \beta_{b_{2}} .
\end{gathered}
$$

Since the relative eddy angular velocity $=2 u_{2} / D_{2}$, then

$$
\Delta W_{u_{2}}=\varepsilon_{2} \frac{\pi u_{2}}{Z} \sin \beta_{b_{2}}, \quad \text { or } \quad \frac{\Delta W_{u_{2}}}{u_{2}}=\varepsilon_{2} \frac{\pi}{Z} \sin \beta_{b_{2}} .
$$

The slip factor is given by

$$
\sigma=\frac{V_{u_{2 \mathrm{act}}}}{V_{u_{2}}}=1-\frac{\Delta V_{u_{2}}}{V_{u_{2}}}=1-\frac{\Delta W_{u_{2}} / u_{2}}{V_{u_{2}} / u_{2}} .
$$

Substituting for $\Delta W_{u_{2}} / u_{2}$ from (16) and for $V_{u_{2}} / u_{2}$ from (8), the formulae proposed by Stodola, cited in [7] for the calculation of slip factor, $\sigma$, are obtained which are

$$
\sigma=1-\frac{\varepsilon_{2}(\pi / Z) \sin \beta_{b_{2}}}{1+\left(V_{r_{2}} / u_{2}\right) \cot \beta_{b_{2}}}=1-\frac{\varepsilon_{2}(\pi / Z) \sin \beta_{b_{2}}}{1+(\psi / \varphi) \cot \beta_{b_{2}}} .
$$

At $\psi=0\left(\sigma=\sigma_{0}\right)$

$$
1-\sigma_{0}=\varepsilon_{2} \frac{\pi}{Z} \sin \beta_{b_{2}}
$$

Therefore,

$$
1-\sigma=\frac{1-\sigma_{0}}{y}
$$

where

$$
y=1+\frac{\psi}{\varphi} \cot \beta_{b_{2}}
$$

Wiesner [8] introduced an empirical expression which extremely well fits the experimental results of slip factor for wide range of practical blade angles and number of blades. It is used in this work and given as

$$
1-\sigma_{0}=\frac{\sqrt{\sin \beta_{b_{2}}}}{Z^{0.7}} \text { for } \frac{D_{1}}{D_{2}} \leq \varepsilon_{\text {limit }},
$$

where $\varepsilon_{\text {limit }}$ is the limiting diameter ratio,

$$
\varepsilon_{\text {limit }}=e^{-\left(8.16 \sin \beta_{b_{2}} / Z\right)} .
$$

It is assumed that the water entering the pump impeller is purely in the radial direction. Relative to the impeller, the fluid has an angular velocity of $-\omega$, relative eddy, and thus the relative flow at blade inlet acquires an additional component $\Delta^{\prime} W_{u_{1}}$ opposite to rotational direction, as seen in Figure 4, which is

$$
\Delta^{\prime} W_{u_{1}}=\omega \frac{d_{1}}{2}
$$

where

$$
\begin{aligned}
d_{1} & =\varepsilon_{1} \frac{\pi D_{1}}{Z} \sin \beta_{b_{1}}, \\
\frac{d_{1}}{D_{2}} & =\varepsilon_{1} \frac{\pi}{Z} \frac{D_{1}}{D_{2}} \sin \beta_{b_{1}} .
\end{aligned}
$$

Hence,

$$
\Delta^{\prime} W_{u_{1}}=\varepsilon_{1} \frac{\pi}{Z} u_{1} \sin \beta_{b_{1}}
$$




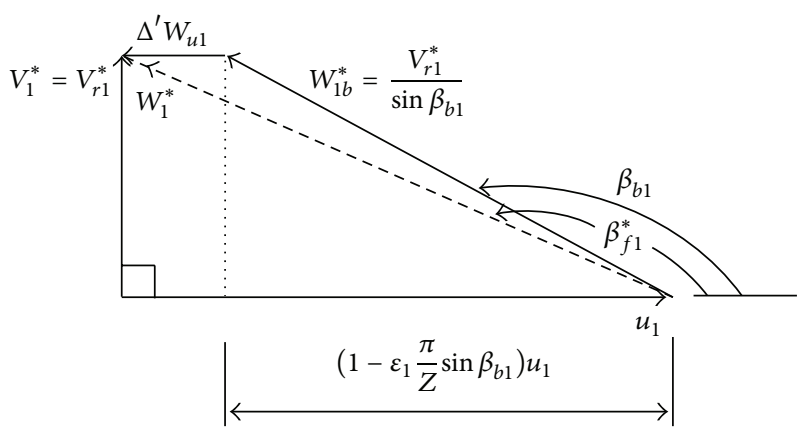

FIGURE 4: Velocity diagram at impeller inlet without shock.

When the pump that operates at a discharge $Q$ differs from that at designed condition $Q^{*}$, the relative flow velocity at blade inlet tends to acquire an additional component in counter of the rotational direction, $\Delta^{\prime \prime} W_{u_{1}}$. So, the flow enters the blade passage tangent to the blade surface, and a shock eddy or a shock circulation exists prior to the blade leading edge inside pump eye. As could be noticed from Figure 5, the relative velocity additional rotational speed at blade inlet equals

$$
\Delta^{\prime \prime} W_{u_{1}}=u_{1}\left(1-\varepsilon_{1} \frac{\pi}{Z} \sin \beta_{b_{1}}\right)-V_{r_{1}} \cot \left(180-\beta_{b_{1}}\right) .
$$

2.4. Euler Equation of Turbomachines. In the case that the fluid entering the pump impeller is purely in the radial direction without swirl, the pump Euler head is given as $[6,7]$

$$
H_{\infty}=\frac{u_{2} V_{u_{2}}}{g} \text {. }
$$

Due to the fluid slippage at impeller exit, the actual head given to fluid by the impeller, $H_{0}$, is calculated from $[6,7]$ :

$$
H_{0}=\frac{u_{2} \cdot V_{u_{2} \text { act }}}{g}=\frac{u_{2} \cdot \sigma V_{u_{2}}}{g}=\sigma \cdot H_{\infty} .
$$

And so, the slippage head loss is

$$
h_{l_{\mathrm{slp}}}=H_{\infty}-H_{0}=(1-\sigma) H_{\infty} .
$$

2.5. Pump Volute. The flow that discharges from the impeller requires careful handling in order to preserve the gains in energy imparted to the fluid. This requires the conversion of velocity head to pressure head by means of a diffuser, and this inevitably implies hydraulic losses. The application of mass conservation to a volute element, [9], reveals that the discharge flow from impeller is matched to the flow in the volute if $d A_{V} / d \theta=V_{r_{3}} / V_{u_{3}} r_{3} b_{3}$. This requires a circumferentially uniform rate of increase of the volute area $A_{V}$ over the entire development of the spiral $(\theta)$. Consequently, for a given impeller, there exist a specific volute angle $\alpha_{V}$ and a specific volute throat inlet area $A_{V_{\text {th }}}$ for the volute geometry.

The volute angle $\alpha_{V}$, Figure 6 , is chosen to match the angle of flow entering the volute $\alpha_{3 \mathrm{eq}}$ at a certain pump operating

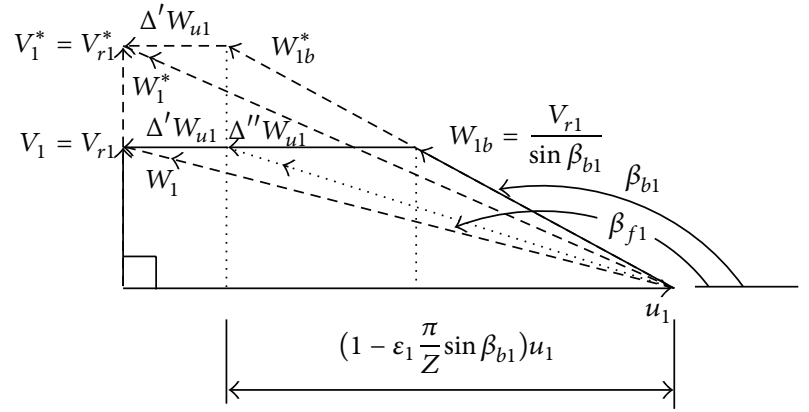

FIGURE 5: Velocity diagram at blade inlet with shock eddy.

point. With a uniform rate of increase of the volute area, the volute angle is

$$
\tan \alpha_{V}=\frac{A_{V_{\mathrm{th}}}}{\pi D_{3} b_{3}}=\frac{A_{V_{\mathrm{th}}}}{\pi D_{2} b_{2}\left(D_{3} / D_{2}\right)\left(b_{3} / b_{2}\right)} .
$$

The volute outlet flow velocity $V_{4}=Q / A_{V_{\text {th }}}$ and the volute outlet flow velocity coefficient $C_{V_{4}}=V_{4} / \sqrt{2 g H}$ are

$$
\begin{gathered}
V_{4}=\frac{\eta_{\mathrm{vol}} \cdot V_{r_{2}} \pi D_{2} b_{2} \varepsilon_{2}}{\pi D_{3} b_{3} \cdot \tan \alpha_{V}}=\frac{\eta_{\mathrm{vol}} \varepsilon_{2}}{\tan \alpha_{V}} \frac{D_{2}}{D_{3}} \frac{b_{2}}{b_{3}} \cdot V_{r_{2}}, \\
C_{V_{4}}=\frac{\varepsilon_{2} \eta_{\mathrm{vol}}}{\left(D_{3} / D_{2}\right)\left(b_{3} / b_{2}\right) \tan \alpha_{V}} \cdot \psi .
\end{gathered}
$$

The throat is assumed to have an expanding angle $\alpha_{\mathrm{th}}$, and hence the throat diameter equals

$$
D_{\text {th }}=\pi D_{3} \tan \alpha_{V}+L_{\text {th }} \tan \alpha_{\text {th }} .
$$

With the assumption that the throat height $L_{\text {th }}=D_{3}$, thus

$$
\frac{D_{\mathrm{th}}}{D_{3}}=\pi \tan \alpha_{V}+\tan \alpha_{\mathrm{th}}
$$

The throat outlet flow velocity $V_{5}=Q / A_{\text {th }}$, and using (2) and (3), then

$$
V_{5}=4 \varepsilon_{2} \eta_{\mathrm{vol}} \frac{D_{2}^{2}}{D_{\mathrm{th}}^{2}} \frac{b_{2}}{D_{2}} \cdot V_{r_{2}} .
$$

The throat outlet flow velocity coefficient $C_{V_{5}}\left(=V_{5} / \sqrt{2 g H}\right)$ equals

$$
C_{V_{5}}=\frac{4 \varepsilon_{2} \eta_{\mathrm{vol}}\left(b_{2} / D_{2}\right)}{\left(D_{\mathrm{th}} / D_{3}\right)^{2}\left(D_{3} / D_{2}\right)^{2}} \cdot \psi
$$

It is assumed that the throat diameter $D_{\text {th }}$ equals the eye diameter $D_{\text {eye }}$; that is, $D_{\text {eye }} / D_{\text {th }}=1$, which equals the suction pipe diameter $D_{s}$. Thus, the throat outlet flow velocity, $V_{5}$, equals the flow velocity at suction pipe, $V_{s}$. Therefore,

$$
V_{5}=V_{s}=\eta_{\mathrm{vol}} \cdot V_{\text {eye }} .
$$

And the eye velocity coefficient is

$$
C_{V_{\text {eye }}}=\frac{V_{\text {eye }}}{\sqrt{2 g H}}=\frac{C_{V_{5}}}{\eta_{\text {vol }}}=\frac{4 \varepsilon_{2}\left(b_{2} / D_{2}\right)}{\left(D_{\text {th }} / D_{3}\right)^{2}\left(D_{3} / D_{2}\right)^{2}} \cdot \psi .
$$



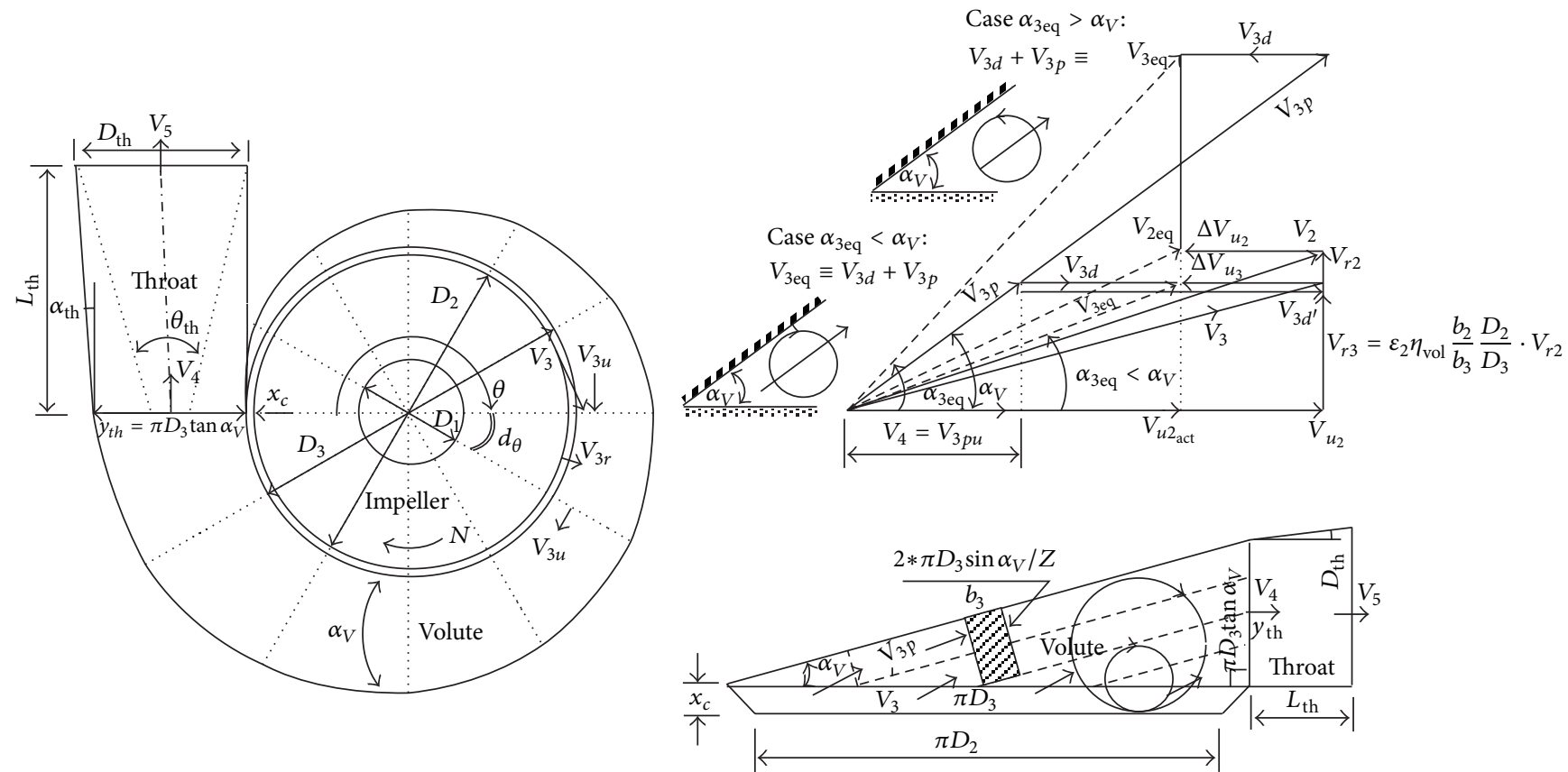

Figure 6: Pump and volute notations.

The eye diameter relative to the impeller inlet diameter is therefore

$$
\frac{D_{\text {eye }}}{D_{1}}=\frac{D_{\text {eye }}}{D_{\text {th }}} \frac{D_{\text {th }}}{D_{3}} \frac{D_{3}}{D_{2}} \frac{D_{2}}{D_{1}}=\frac{\left(D_{3} / D_{2}\right)\left(\pi \tan \alpha_{V}+\tan \alpha_{\mathrm{th}}\right)}{\left(D_{1} / D_{2}\right)} .
$$

The ratio $\left(D_{\text {eye }} / D_{1}\right)$ must not exceed 1 , and thus an upper limit is imposed on the volute angle:

$$
\tan \alpha_{V}<\frac{\left(D_{1} / D_{2}\right)}{\pi\left(D_{3} / D_{2}\right)}-\frac{\tan \alpha_{\mathrm{th}}}{\pi} .
$$

The throat has a cone angle $\theta_{\text {th }}$, where

$$
\tan \left(\frac{\theta_{\mathrm{th}}}{2}\right)=\frac{\left(D_{\mathrm{th}}-b_{3}\right) / 2}{L_{\mathrm{th}}} .
$$

Substituting from (35),

$$
\tan \left(\frac{\theta_{\mathrm{th}}}{2}\right)=\frac{1}{2}\left[\pi \tan \alpha_{V}+\tan \alpha_{\mathrm{th}}-\frac{\left(b_{3} / b_{2}\right)\left(b_{2} / D_{2}\right)}{\left(D_{3} / D_{2}\right)}\right] .
$$

2.6. Volute Loss Model. Prediction models that account for the main features of the swirling flow in volutes, reviewed in [4], do not account for the circulatory flow initiated in volute at off-design discharge operation of pump. In this work, a model is proposed to account the volute head loss at offdesign pump operation.

The flow enters the volute with a through-velocity $V_{3}$ at an angle $\alpha_{3}$ (which may differ from that of the volute angle $\alpha_{V}$ ) on which an eddy of a tangential velocity $\Delta V_{u_{3}}=$ $\Delta V_{u_{2}}$ is superimposed opposite to impeller motion. This inlet volute velocity $V_{3}$ is decomposed into a velocity parallel to the direction of volute, $V_{3 p}$, and another one in the tangential direction of impeller, $V_{3 d}^{\prime}$ (Figure 6). The velocity component $V_{3 d}^{\prime}$ is the motive of a second circulatory motion given to the volute flow in direction of impeller motion. Thus, the net circulation velocity of flow in volute is $V_{3 d}=V_{3 d}^{\prime}-\Delta V_{u_{3}}$ in direction of impeller motion. The component of the velocity $V_{3 p}$ in the tangential direction denoted as $V_{3 p u}$ equals the volute outlet velocity $V_{4}$.

The volute loss model counts friction loss associated with the volute throw flow velocity, diffusion friction loss due to circulation associated with volute flow, loss due to vanishing of radial flow at volute outlet, and loss inside pump volute throat. Consequently, the volute head loss and the volute head loss relative to the pump head are written, respectively, as

$$
\begin{gathered}
h_{l_{V}}=C_{f_{V}} \frac{V_{3 p}^{2}}{2 g}+C_{d_{V}} \frac{V_{3 d}^{2}}{2 g}+\frac{V_{3 r}^{2}}{2 g}+h_{l_{\mathrm{th}}}, \\
\frac{h_{l_{V}}}{H}=C_{f_{V}} \cdot C_{V_{3 p}}^{2}+C_{d_{V}} \cdot C_{V_{3 d}}^{2}+C_{V_{3 r}}^{2}+C_{f_{\mathrm{th}}} \cdot C_{V_{4}}^{2},
\end{gathered}
$$

where $C_{f_{V}}$ is the volute friction loss coefficient:

$$
C_{f_{V}}=f_{V} \frac{L_{V}}{D_{h_{V}}}=f_{V}\left(\frac{L_{V}}{D_{2}}\right)\left(\frac{1}{D_{h_{V}} / D_{2}}\right),
$$

where $f_{V}$ is the volute friction coefficient, $D_{h_{V}}$ is the volute hydraulic diameter, and $L_{V}$ is the average volute length:

$$
\begin{gathered}
L_{V}=\frac{1}{2} \frac{\pi D_{3}}{\cos \alpha_{V}}, \\
\frac{L_{V}}{D_{2}}=\frac{1}{2} \frac{\pi}{\cos \alpha_{V}}\left(\frac{D_{3}}{D_{2}}\right) .
\end{gathered}
$$


The average volute hydraulic diameter relative to impeller diameter is

$$
\frac{1}{D_{h_{V}} / D_{2}}=\frac{1}{2\left(b_{3} / b_{2}\right)\left(b_{2} / D_{2}\right)}+\frac{1}{8(\pi / Z)\left(D_{3} / D_{2}\right) \sin \alpha_{V}} .
$$

The volute friction coefficient $f_{V}$ which corresponds to a pipe flow is function of the volute Reynolds number $\operatorname{Re}_{V}$ and the roughness $\kappa,[10]$ :

$$
f_{V}=\frac{0.3086}{\left\{\log \left[\left(6.9 / \operatorname{Re}_{V}\right)+\left(\left(\kappa / D_{h_{V}}\right) / 3.7\right)^{1.11}\right]\right\}^{2}}
$$

where

$$
\frac{\kappa}{D_{h_{V}}}=\frac{\left(\kappa / D_{2}\right)}{\left(D_{h_{V}} / D_{2}\right)}
$$

The volute Reynolds number is calculated as

$$
\operatorname{Re}_{V}=\frac{V_{3 p} \cdot D_{h_{V}}}{\nu}=2 \frac{C_{V_{3 p}}}{\varphi}\left(\frac{D_{h_{V}}}{D_{2}}\right) \cdot \mathrm{Re}_{2},
$$

where

$$
\operatorname{Re}_{2}=\frac{u_{2} \cdot D_{2} / 2}{v}
$$

In the first term of (44) and (45), $V_{3 p}$ is the volute throw flow velocity (Figure 6) and given as

$$
V_{3 p}=\frac{V_{4}}{\cos \alpha_{V}}, \quad \text { or } \quad C_{V_{3 p}}=\frac{C_{V_{4}}}{\cos \alpha_{V}} .
$$

In the second term of (44) and (45), $C_{d_{V}}$ is the volute diffusion loss coefficient which could be assumed to have the value $C_{d_{V}}=0.8$, and $V_{3 d}$ is the volute circulatory velocity component:

$$
\begin{gathered}
V_{3 d}=V_{u_{2 \mathrm{act}}}-V_{4} \\
C_{V_{3 d}}=\frac{V_{3 d}}{\sqrt{2 g H}}=\sigma\left(\varphi+\psi \cot \beta_{b_{2}}\right)-C_{V_{4}} .
\end{gathered}
$$

The third term of (45) is

$$
C_{V_{3 r}}=\frac{V_{3 r}}{\sqrt{2 g H}}=\frac{\varepsilon_{2} \eta_{\mathrm{vol}}}{\left(D_{3} / D_{2}\right)\left(b_{3} / b_{2}\right)} \cdot \psi .
$$

In the fourth term of (44) and (45), the volute throat head loss $h_{l_{\mathrm{th}}}$ could be calculated as

$$
h_{l_{\mathrm{th}}}=C_{f_{\mathrm{th}}} \frac{V_{4}^{2}}{2 g},
$$

where $C_{f_{\text {th }}}$ is the volute throat friction loss coefficient assumed to be, [10],

$$
C_{f_{\text {th }}}=0.5+2.6 * \sin \left(\frac{\theta_{\text {th }}}{2}\right),
$$

where $\theta_{\text {th }}$ is the throat cone angle.
2.7. Pump Eye Head Loss $h_{l_{\text {eye }}}$. The head loss in pump eye $h_{l_{\text {eye }}}$, [2], and the eye head loss relative to the pump head are

$$
\begin{aligned}
& h_{l_{\text {eye }}}=C_{\text {eye }} \frac{V_{\text {eye }}^{2}}{2 g}, \\
& \frac{h_{l_{\text {eye }}}}{H}=C_{\text {eye }} \frac{V_{\text {eye }}^{2}}{2 g H}=C_{\text {eye }} \cdot C_{V_{\text {eye }}}^{2},
\end{aligned}
$$

where $V_{\text {eye }}$ is the flow velocity in pump eye and $C_{\text {eye }}$ is the eye loss coefficient defined by (39).

2.8. Pump Manometric Head H. The pump manometric head is the difference in static pressure heads between pump outlet and pump eye:

$$
H=\frac{p_{5}-p_{\text {eye }}}{\gamma} .
$$

With the assumption that the throat diameter $D_{\text {th }}$ equals the eye diameter $D_{\text {eye }}$, the flow velocity is the same at pump suction pipe and pump delivery pipe $\left(V_{s}=V_{5}\right)$ and neglecting the difference in elevation head across the pump, then

$$
H=H_{0}-h_{l_{V}}-h_{l_{\text {eye }}}
$$

or

$$
H=H_{\infty}-h_{l_{\text {slp }}}-h_{l_{V}}-h_{l_{\text {eye }}} .
$$

Define a parameter $x$ as

$$
\begin{aligned}
& x=\frac{H}{H_{0}}=\frac{1}{\left[1+\left(h_{l_{V}} / H\right)+\left(h_{l_{\text {eye }}} / H\right)\right]}, \\
& \text { or } \frac{1}{x}=1+\frac{h_{l_{V}}}{H}+\frac{h_{l_{\text {eye }}}}{H} .
\end{aligned}
$$

Using (45) as well as (57) and (61) then

$$
\begin{aligned}
\frac{1}{x}= & 1+C_{f_{V}} \cdot C_{V_{3 p}}^{2}+C_{d_{V}} \cdot C_{V_{3 d}}^{2}+C_{V_{3 r}}^{2}+C_{f_{\mathrm{th}}} \cdot C_{V_{4}}^{2} \\
& +C_{\text {eye }} \cdot C_{V_{\text {eye }}}^{2} .
\end{aligned}
$$

The sum of volute and eye head losses, from (59), is written as

$$
h_{l_{V}}+h_{l_{\text {eye }}}=(1-x) H_{0}=(1-x) \sigma H_{\infty} .
$$

Using (9), (29), and (59), the pump manometric head $H$ is

$$
\begin{aligned}
& H=x \sigma H_{\infty}=x \sigma \frac{u_{2} \cdot V_{u_{2}}}{g}, \\
& H=x \sigma \frac{u_{2}^{2}}{g}\left(1+\frac{\psi}{\varphi} \cot \beta_{b_{2}}\right) .
\end{aligned}
$$

Coefficients. There are two additional groups of coefficients, namely, the pump head coefficients and head loss coefficients. 
The pump head coefficients are the pump manometric head coefficient, $C_{H}$, Euler head coefficient, $C_{H_{\infty}}$, and the head coefficient at impeller outlet, $C_{H_{0}}$. They are defined and given, respectively, as

$$
\begin{gathered}
C_{H}=\frac{H}{u_{2}^{2} / g}=x \sigma\left(1+\frac{\psi}{\varphi} \cot \beta_{b_{2}}\right), \\
C_{H_{\infty}}=\frac{H_{\infty}}{u_{2}^{2} / g}=1+\frac{\psi}{\varphi} \cot \beta_{b_{2}}, \\
C_{H_{0}}=\frac{H_{0}}{u_{2}^{2} / g}=\sigma\left(1+\frac{\psi}{\varphi} \cot \beta_{b_{2}}\right) .
\end{gathered}
$$

According to (21) and (65b), $y=C_{H_{\infty}}$.

The head loss coefficients include the slippage head loss coefficient, $C_{h_{l_{\text {slp }}}}$, the volute-eye head loss coefficient, $C_{h_{l_{V+e y e}}}$, the eye head loss coefficient, $C_{h_{\text {leye }}}$, and the volute head loss coefficient, $C_{h_{l_{V}}}$. They are given, respectively, as

$$
\begin{gathered}
C_{h_{l_{\text {slp }}}}=\frac{h_{l_{\text {slp }}}}{u_{2}^{2} / g}=(1-\sigma) C_{H_{\infty}}, \\
C_{h_{l_{V+\text { eye }}}}=\frac{h_{l_{V}}+h_{l_{\text {eye }}}}{u_{2}^{2} / g}=(1-x) \sigma C_{H_{\infty}}, \\
C_{h_{\text {leye }}}=\frac{h_{l_{\text {eye }}}}{u_{2}^{2} / g}=C_{\text {eye }} \cdot C_{V_{\text {eye }}}^{2} \cdot \frac{1}{2 \varphi^{2}}, \\
C_{h_{l_{V}}}=\frac{h_{l_{V}}}{u_{2}^{2} / g}=\left(C_{f_{V}} \cdot C_{V_{3 p}}^{2}+C_{d_{V}} \cdot C_{V_{3 d}}^{2}+C_{V_{3 r}}^{2}\right. \\
\left.\quad+C_{f_{\mathrm{th}}} \cdot C_{V_{4}}^{2}\right) \cdot \frac{1}{2 \varphi^{2}} .
\end{gathered}
$$

The relationship between the pump speed coefficient $\varphi$ and the pump flow coefficient $\psi$ is derived as follows.

Using (11), (64) becomes

$$
H=x \sigma \frac{u_{2}^{2}}{g} \frac{\cot \alpha_{2}}{\cot \alpha_{2}-\cot \beta_{b_{2}}} .
$$

Dividing both sides by $H$, noting that $u_{2}^{2} /(2 g H)=\varphi^{2}$, and using (11), (64) becomes a quadric equation for $\cot \alpha_{2}$ :

$$
\cot ^{2} \alpha_{2}-\cot \beta_{b_{2}} \cdot \cot \alpha_{2}-\frac{1}{2 x \sigma \psi^{2}}=0,
$$

which has a solution (since $\cot \alpha_{2}$ should be greater than $\cot \beta_{b_{2}}$, whence only the +ve sign is considered)

$$
\cot \alpha_{2}=\frac{1}{2} \cot \beta_{b_{2}}+\frac{1}{2} \sqrt{\cot ^{2} \beta_{b_{2}}+\frac{2}{x \sigma \psi^{2}}} .
$$

Multiplying (68) by $\psi$ and then subtracting $\psi \cot \beta_{b_{2}}$ from both sides and using (11) yield the following relation for $\varphi$ :

$$
\varphi=-\frac{1}{2} \psi \cot \beta_{b_{2}}+\frac{1}{2} \sqrt{\psi^{2} \cot ^{2} \beta_{b_{2}}+\frac{2}{x \sigma}} .
$$

2.9. Pump Shaft Power $P_{\mathrm{sh}}$, and Pump Shaft Head $H_{\mathrm{sh}}$. The total shaft power required to drive the impeller is

$$
P_{\mathrm{sh}}=P_{\mathrm{sh}_{0}}^{\prime}+P_{l_{f}}^{\prime}+P_{l_{\mathrm{cirin}}}^{\prime}+P_{l_{D}}
$$

where $P_{\mathrm{sh}_{0}}^{\prime}=\gamma\left(Q+Q_{L}\right) H_{0}$ is the impeller power given to water, $P_{l_{f}}^{\prime}=\gamma\left(Q+Q_{L}\right) h_{l_{f}}$ is the power lost in friction inside impeller, $P_{l_{\text {cirin }}}^{\prime}=\gamma\left(Q+Q_{L}\right) h_{l_{\text {cir }} \text { in }}$ is the power needed for given circulation to flow at impeller inlet, and $P_{l_{\mathrm{D}}}$ is the power lost in friction on outside surface of impeller disks.

The total shaft power and pump shaft head can be simplified, respectively, to

$$
P_{\mathrm{sh}}=P_{\mathrm{sh}_{0}}+P_{l_{f}}+P_{l_{\mathrm{cirin}}}+P_{l_{\mathrm{vol}}}+P_{l_{D}}
$$

in which

$$
\begin{aligned}
& P_{\mathrm{sh}_{0}}=\gamma Q H_{0}, \quad P_{l_{f}}=\gamma Q h_{l_{f}}, \quad P_{l_{\text {cirin }}}=\gamma Q h_{l_{\text {cirin }_{\text {in }}}}, \\
& P_{l_{\mathrm{vol}}}=\gamma Q h_{l_{\mathrm{vol}}}=\gamma Q_{L}\left(H_{0}+h_{l_{f}}+h_{l_{\mathrm{cir} \text { in }}}\right), \\
& P_{l_{D}}=\gamma Q h_{l_{D}}
\end{aligned}
$$

where $h_{l_{f}}$ is the impeller skin friction head loss, $h_{l_{\text {cirin }}}$ is the inlet shock circulation head loss, $h_{l_{\text {yol }}}$ is the volumetric (leakage) head loss, and $h_{l_{D}}$ is the disk friction head loss.

The shaft head $H_{\mathrm{sh}}=P_{\mathrm{sh}} /(\gamma \mathrm{Q})$ and the shaft head coefficient $C_{H_{\mathrm{sh}}}=H_{\mathrm{sh}} /\left(u_{2}^{2} / g\right)$ are given, respectively, as

$$
\begin{gathered}
H_{\mathrm{sh}}=H_{0}+h_{l_{f}}+h_{l_{\mathrm{cirin}}}+h_{l_{\mathrm{vol}}}+h_{l_{D}}, \\
C_{H_{\mathrm{sh}}}=C_{H_{0}}+C_{h_{l_{f}}}+C_{h_{l \mathrm{cirin}}}+C_{h_{l \mathrm{vol}}}+C_{h_{l D}},
\end{gathered}
$$

where $C_{h_{l_{f}}}=h_{l_{f}} /\left(u_{2}^{2} / g\right)$ is the impeller skin friction head loss coefficient, $C_{h_{\text {lciri }}}=h_{l_{\text {ciri }}} /\left(u_{2}^{2} / g\right)$ is the inlet shock circulation head loss coefficient, $C_{h_{l v 0 l}}=h_{l_{\text {vol }}} /\left(u_{2}^{2} / g\right)$ is the volumetric head loss coefficient, and $C_{h_{l D}}=h_{l_{D}} /\left(u_{2}^{2} / g\right)$ is the disk friction head loss coefficient.

2.10. Pump Efficiency (Manometric Efficiency) $\eta$. The pump manometric efficiency is the ratio of gained water power $\left(P_{w}\right)$ to the pump shaft power $\left(P_{\mathrm{sh}}\right)$ supplied to pump impeller. According to its definition, it takes the following forms:

$$
\begin{aligned}
& \eta=\frac{P_{w}}{P_{\mathrm{sh}}}=\frac{P_{w}}{P_{\mathrm{sh}_{0}}+P_{l_{f}}+P_{\mathrm{cirin}}+P_{l_{\mathrm{vol}}}+P_{l_{D}}}, \\
& \eta=\frac{H}{H_{\text {sh }}}=\frac{H_{\infty}-h_{l_{\text {sp }}}-h_{l_{V}}-h_{l_{\text {eye }}}}{H_{0}+h_{l_{f}}+h_{l_{\text {cirin }}}+h_{l_{\text {vol }}}+h_{l_{D}}}, \\
& \eta=\frac{C_{H}}{C_{H_{s h}}}=\frac{C_{H_{\infty}}-C_{h_{l s p}}-C_{h_{l V+\text { eye }}}}{C_{H_{0}}+C_{h_{l_{f}}}+C_{h_{l \text { cri }}}+C_{h_{l \text { vol }}}+C_{h_{l D}}} .
\end{aligned}
$$


2.11. Pump Shaft Power Coefficient $C_{P_{s h}}$. The pump shaft power coefficient and the pump water power coefficient are given, respectively, as

$$
\begin{gathered}
C_{P_{\mathrm{sh}}}=\frac{P_{\mathrm{sh}}}{\rho(N / 60)^{3} D_{2}^{5}}=\pi^{2} C_{\mathrm{Q}} C_{H_{\mathrm{sh}}}, \\
C_{P_{w}}=\frac{P_{w}}{\rho(N / 60)^{3} D_{2}^{5}}=\pi^{2} C_{\mathrm{Q}} C_{H} .
\end{gathered}
$$

2.12. Impeller Skin Friction Power Head Loss $h_{l_{f}}$. The impeller hydraulic friction head loss $h_{l_{f}}$ is estimated by the theory of flow through pipes and is given by [11]:

$$
h_{l_{f}}=4 C_{d_{i}} \frac{L_{b}}{D_{\text {hyd }}} \frac{W_{\mathrm{av}}^{2}}{2 g},
$$

where $C_{d_{i}}$ is the dissipation coefficient, $L_{b}$ is the blade length, $D_{\text {hyd }}$ is the hydraulic diameter, and $W_{\mathrm{av}}$ is the average relative velocity. Therefore, the impeller skin friction head loss coefficient could be calculated from

$$
C_{h_{l f}}=\frac{h_{l_{f}}}{u_{2}^{2} / g}=4 C_{d_{i}} \frac{\left(L_{b} / D_{2}\right)}{\left(D_{\text {hyd }} / D_{2}\right)} \frac{1}{2}\left(\frac{W_{\mathrm{av}}}{u_{2}}\right)^{2} .
$$

The hydraulic diameter and the average relative velocity are given, respectively, as, Gülich [11]

$$
\begin{gathered}
D_{\text {hyd }}=\frac{4 * \text { Area }}{\text { Perimeter }}=\frac{2\left(d_{1} b_{1}+d_{2} b_{2}\right)}{d_{1}+b_{1}+d_{2}+b_{2}}, \\
W_{\mathrm{av}}=\frac{Q_{i} / Z}{A_{\mathrm{av}}}=\frac{Q_{i} / Z}{\left(d_{1} b_{1}+d_{2} b_{2}\right) / 2} .
\end{gathered}
$$

Substituting for $d_{1},(26), d_{2},(15)$, and $Q_{i}$, (3), yield:

$$
\begin{gathered}
\frac{D_{\text {hyd }}}{D_{2}}=\frac{2\left(\left(d_{1} / D_{2}\right)\left(b_{1} / b_{2}\right)\left(b_{2} / D_{2}\right)+\left(d_{2} / D_{2}\right)\left(b_{2} / D_{2}\right)\right)}{\left(d_{1} / D_{2}\right)+\left(b_{1} / b_{2}\right)\left(b_{2} / D_{2}\right)+\left(d_{2} / D_{2}\right)+\left(b_{2} / D_{2}\right)}, \\
\frac{W_{\mathrm{av}}}{u_{2}}=\frac{\varepsilon_{2}(\pi / Z)\left(b_{2} / D_{2}\right)}{1 / 2\left(\left(d_{1} / D_{2}\right)\left(b_{1} / b_{2}\right)\left(b_{2} / D_{2}\right)+\left(d_{2} / D_{2}\right)\left(b_{2} / D_{2}\right)\right)} \cdot \frac{\psi}{\varphi} .
\end{gathered}
$$

The blade length is $L_{b}=\left((1 / 2)\left(D_{2}-D_{1}\right)\right) / \sin \beta_{\mathrm{bm}}$, and thus

$$
\frac{L_{b}}{D_{2}}=\frac{1}{2}\left(1-\frac{D_{1}}{D_{2}}\right) \frac{1}{\sin \beta_{\mathrm{bm}}}
$$

where

$$
\beta_{\mathrm{bm}}=\frac{\left(\beta_{b_{1}}+\beta_{b_{2}}\right)}{2}
$$

The impeller dissipation coefficient is given as, Gülich [11]

$$
4 C_{d_{i}}=\left(f_{i}+0.006\right)\left(1.1+4 \frac{b_{2}}{D_{2}}\right) .
$$

The impeller friction coefficient $f_{i}$ is function of the average impeller Reynolds number Re and the roughness $\kappa$ [10]:

$$
f_{i}=\frac{0.3086}{\left\{\log \left[(6.9 / \mathrm{Re})+\left(\left(\kappa / D_{\text {hyd }}\right) / 3.7\right)^{1.11}\right]\right\}^{2}}
$$

where

$$
\begin{gathered}
\frac{\kappa}{D_{\text {hyd }}}=\frac{\left(\kappa / D_{2}\right)}{\left(D_{\text {hyd }} / D_{2}\right)} . \\
\operatorname{Re}=\frac{W_{\text {av }} \cdot D_{\text {hyd }}}{\nu}=2\left(\frac{W_{\text {av }}}{u_{2}}\right)\left(\frac{D_{\text {hyd }}}{D_{2}}\right) \cdot \operatorname{Re}_{2},
\end{gathered}
$$

where $\mathrm{Re}_{2}$ is defined by (53).

2.13. Disk Friction Head Loss $h_{l_{D}}$. The disk friction power loss $P_{l_{D}}$ is the power loss in the fluid between external surfaces of the impeller disks and internal walls of the pump casing, Figure 7. The $P_{l_{D}}$ can be estimated as

$$
P_{l_{D}}=2 * \int_{0}^{r_{2}} \omega \cdot r \cdot \tau \cdot 2 \pi r d r
$$

with

$$
\tau=\frac{f_{D}}{4} \cdot \frac{1}{2} \rho u^{2}=\frac{f_{D}}{4} \cdot \frac{1}{2} \rho(\omega r)^{2},
$$

where $\tau$ is the shear stress in circumferential direction and $f_{D}$ is the disk skin friction coefficient, and it is assumed constant along the disk surface.

Thus,

$$
P_{l_{D}}=\frac{\pi}{2} \rho \omega^{3} \int_{0}^{r_{2}} f_{D} r^{4} d r
$$

Therefore, the final expression after the integration for the disk friction power loss and, consequently, the impeller disk friction head loss are

$$
\begin{gathered}
P_{l_{D}}=\frac{\pi}{2} f_{D} \rho \omega^{3} \frac{r_{2}^{5}}{5}=\frac{\pi}{40} f_{D} \rho u_{2}^{3} D_{2}^{2}, \\
h_{l_{D}}=\frac{P_{l_{D}}}{\rho g Q}=\frac{\pi}{40} \frac{f_{D}}{g} \frac{u_{2}^{3} D_{2}^{2}}{Q} .
\end{gathered}
$$

The impeller disk friction head loss coefficient $C_{h_{l_{D}}}$ is given as

$$
C_{h_{l_{D}}} \equiv \frac{h_{l_{D}}}{u_{2}^{2} / g}=K_{D} \cdot \frac{1}{\eta_{\mathrm{vol}}} \frac{\varphi}{\psi},
$$

where $K_{D}$ is the impeller disk loss coefficient,

$$
K_{D}=\frac{1}{40} \frac{f_{D}}{\varepsilon_{2}\left(b_{2} / D_{2}\right)} .
$$

It is derived by substituting for $Q$ from (2) and (3) and using the definition of $\psi$ which yields $Q=\eta_{\mathrm{vol}} \varepsilon_{2} \cdot \psi \sqrt{2 g H} \cdot \pi D_{2} b_{2}$. 
Correlations for $f_{D}$ were obtained by Kruyt, cited in [12]. Four different regimes were identified, Figure 8: Regime I (laminar flow, boundary layers have merged), Regime II (laminar flow with two separate boundary layers), Regime III (turbulent flow, boundary layers have merged), and Regime IV (turbulent flow with two separate boundary layers). These regimes are characterized by the Reynolds number, $\mathrm{Re}_{2}=$ $u_{2} \cdot\left(D_{2} / 2\right) / v$, and a nondimensional gap parameter, $G=$ $y_{0} /\left(D_{2} / 2\right)$, where $y_{0}$ is the axial gap between impeller disk and casing (Figure 7). The equations of curves 1 to 5 in Figure 8 are $G=1.62 \operatorname{Re}_{2}^{-5 / 11}, G=188 \operatorname{Re}_{2}^{-9 / 10}, G=$ $0.57 * 10^{-6} \operatorname{Re}_{2}^{15 / 16}, \operatorname{Re}=1.58 * 10^{5}$, and $G=0.402 \mathrm{Re}_{2}^{-3 / 16}$, respectively. The disk friction coefficient for each regime is given as

$$
\begin{aligned}
& \text { Regime I }: f_{D}=10 G^{-1} \mathrm{Re}_{2}^{-1} \\
& \text { Regime II }: f_{D}=\frac{18.5}{\pi} G^{1 / 10} \operatorname{Re}_{2}^{-1 / 2} \\
& \text { Regime III }: f_{D}=\frac{0.4}{\pi} G^{-1 / 6} \mathrm{Re}_{2}^{-1 / 4} \\
& \text { Regime IV }: f_{D}=\frac{0.51}{\pi} G^{1 / 10} \mathrm{Re}_{2}^{-1 / 5} .
\end{aligned}
$$

2.14. Volumetric Head Loss $h_{l_{v 01}}$. The volumetric (leakage) head loss and the volumetric head loss coefficient are given as follows after using (2):

$$
\begin{aligned}
& h_{l_{\mathrm{vol}}}=\frac{P_{l_{\mathrm{vol}}}}{\gamma Q}=\frac{Q_{L}}{Q}\left(H_{0}+h_{l_{f}}+h_{l_{\mathrm{cirin}}}\right) \\
&=\left(\frac{1}{\eta_{\mathrm{vol}}}-1\right) \cdot\left(H_{0}+h_{l_{f}}+h_{l_{\mathrm{cirin}}}\right) \\
& C_{h_{l_{\mathrm{vol}}}=} \frac{h_{l_{\mathrm{vol}}}}{u_{2}^{2} / g}=\left(\frac{1}{\eta_{\mathrm{vol}}}-1\right) \cdot\left(C_{H_{0}}+C_{h_{l f}}+C_{h_{l_{\mathrm{cir}} \mathrm{in}}}\right) .
\end{aligned}
$$

The leakage flow rate $Q_{L}$ can be estimated using orifice formula [13]:

$$
Q_{L}=C_{\mathrm{dL}} \cdot a_{c} \sqrt{2 g \Delta H_{c}},
$$

where $a_{c}$ is the clearance area of wearing ring $\left(=\pi D_{\text {eye }} y_{c}\right), y_{c}$ is the clearance of wearing ring, Figure $7, C_{\mathrm{dL}}$ is the leakage discharge coefficient $(\approx 0.6)$, and $\Delta H_{c}$ is the pressure head drop across the clearance:

$$
\Delta H_{c}=\frac{p_{c}-p_{s}}{\gamma}=\frac{p_{3}-p_{s}}{\gamma}-\frac{p_{3}-p_{c}}{\gamma},
$$

where $p_{c}$ is the pressure before clearance and $p_{s}$ is the pressure after clearance at suction side of the impeller (Figure 7).

The pressure head difference between volute and pump suction side equals

$$
\begin{aligned}
\frac{p_{3}-p_{s}}{\gamma} & =\frac{p_{5}-p_{s}}{\gamma}+\frac{p_{3}-p_{5}}{\gamma} \\
& =H+\left[h_{l_{V}}-\left(\frac{V_{4}^{2}}{2 g}-\frac{V_{5}^{2}}{2 g}\right)\right] \\
& =H_{0}-h_{l_{\text {eye }}}-\left(\frac{V_{4}^{2}}{2 g}-\frac{V_{5}^{2}}{2 g}\right) .
\end{aligned}
$$

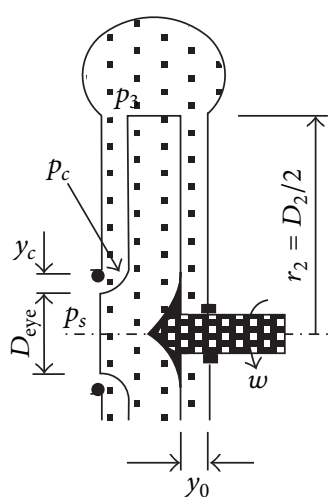

FIGURE 7: Disk and wearing ring clearances.

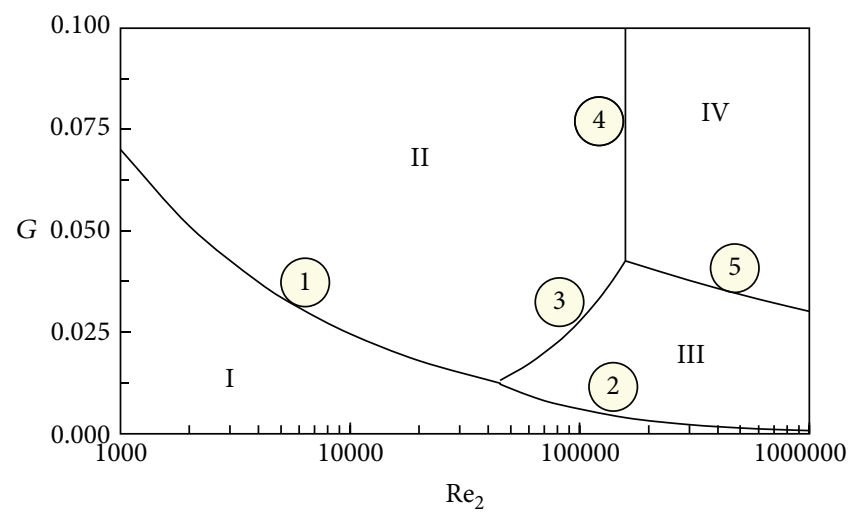

Figure 8: Disk friction flow regimes [12].

The pressure distribution along the radial direction of impeller shroud is parabolic [13], and thus the pressure head difference between impeller outlet and before clearance is given by

$$
\frac{p_{3}-p_{c}}{\gamma}=\frac{u_{2}^{2}}{8 g}\left(1-\frac{D_{\text {eye }}^{2}}{D_{2}^{2}}\right) .
$$

Thus, the leakage flow rate and the leakage flow rate coefficient, $C_{Q_{L}}$, are

$$
\begin{aligned}
Q_{L}= & C_{\mathrm{dL}} \cdot \pi D_{\text {eye }} y_{c} \cdot u_{2} \sqrt{2} \\
& *\left(C_{H_{0}}-\frac{C_{V_{4}}^{2}-C_{V_{5}}^{2}+C_{\text {eye }} \cdot C_{V_{\text {eye }}}^{2}}{2 \varphi^{2}}\right. \\
& \left.-\frac{1}{8}\left(1-\frac{D_{\text {eye }}^{2}}{D_{2}^{2}}\right)\right)^{1 / 2}, \\
C_{\mathrm{Q}_{L}}= & \frac{Q_{L}}{(N / 60) D_{2}^{3}}=\frac{y_{c}}{D_{\text {eye }}} \frac{\sqrt{2} \pi^{2} C_{\mathrm{dL}}}{\left(D_{2} / D_{\text {eye }}\right)^{2}}
\end{aligned}
$$




$$
\begin{gathered}
*\left(C_{H_{0}}-\frac{C_{V_{4}}^{2}-C_{V_{5}}^{2}+C_{\text {eye }} \cdot C_{V_{\text {eye }}}^{2}}{2 \varphi^{2}}\right. \\
\left.-\frac{1}{8}\left(1-\frac{D_{\text {eye }}^{2}}{D_{2}^{2}}\right)\right)^{1 / 2} .
\end{gathered}
$$

Using (2), $Q_{L} / Q=C_{Q_{L}} / C_{Q}$, and (8) for $C_{Q}$, the pump volumetric efficiency can be deduced after some mathematical manipulations:

$$
\begin{aligned}
\eta_{\text {vol }}=1 & -\left(\frac{y_{c}}{D_{\text {eye }}}\right)\left(\frac{D_{\text {eye }}}{D_{1}}\right)^{2}\left(\frac{D_{1}}{D_{2}}\right)^{2} \frac{\sqrt{2} C_{\text {dL }}}{\left(b_{2} / D_{2}\right) \varepsilon_{2}} \frac{\varphi}{\psi} \\
* & \left(C_{H_{0}}-\frac{C_{V_{4}}^{2}-C_{V_{5}}^{2}+C_{\text {eye }} \cdot C_{V_{\text {eye }}}^{2}}{2 \varphi^{2}}\right. \\
& \left.-\frac{1}{8}\left[1-\left(\frac{D_{\text {eye }}}{D_{1}}\right)^{2}\left(\frac{D_{1}}{D_{2}}\right)^{2}\right]\right)^{1 / 2} .
\end{aligned}
$$

2.15. Inlet Shock Circulation Head Loss $h_{l_{\text {cir }_{\text {in }}}}$. At impeller inlet, a shock circulation exists inside pump eye when the flow discharge differs from that of designed one. As discussed before in Section 2.3, the tangential velocity responsible for this shock eddy is $\Delta^{\prime \prime} W_{u_{1}}$, which could be estimated from (28).

Therefore, the inlet shock circulation head loss equals

$$
h_{l_{\text {iri }}}=\frac{\Delta^{\prime \prime} W_{u_{1}} \cdot u_{1}}{g} .
$$

Thus, the inlet shock circulation head loss coefficient is

$$
\begin{aligned}
C_{h_{l_{\text {cir }}}} & =\frac{h_{l_{\text {cir }}}}{u_{2}^{2} / g} \\
& =\left(1-\varepsilon_{1} \frac{\pi}{Z} \sin \beta_{b_{1}}\right)\left(\frac{D_{1}}{D_{2}}\right)^{2}-\frac{\cot \left(180-\beta_{b_{1}}\right)}{\left(b_{1} / b_{2}\right)\left(\varepsilon_{1} / \varepsilon_{2}\right)} \cdot \frac{\psi}{\varphi} .
\end{aligned}
$$

2.16. Required Net Positive Suction Head $\mathrm{NPSH}_{R}$. The pump net positive suction head NPSH is defined as the difference between the fluid inlet stagnation pressure head and vapour pressure head [13]:

$$
\mathrm{NPSH}=\left(\frac{p_{1}}{\gamma}+\frac{V_{1}^{2}}{2 g}\right)-\frac{p_{v}}{\gamma},
$$

where $p_{1}, V_{1}$ are the absolute pressure and absolute velocity of fluid at impeller inlet and $p_{v}$ is the absolute vapour pressure of fluid at the corresponding fluid temperature.

In the vicinity of the leading edge of the impeller blades, the fluid has to accelerate in order to follow the rotating movement of the blades. This acceleration leads to a drop of the static pressure, which results in a local minimum pressure at blade inlet:

$$
\frac{p_{\min }}{\gamma}=\frac{p_{1}}{\gamma}-\left(\frac{W_{1 b}^{2}}{2 g}-\frac{V_{1}^{2}}{2 g}\right)
$$

Therefore, the pump required net positive suction head is

$$
\mathrm{NPSH}_{R}=\frac{\left(p_{\min }-p_{v}\right)}{\gamma}+\frac{W_{1 b}^{2}}{2 g} .
$$

The pump $\mathrm{NPSH}_{R}$ coefficient is defined as

$$
C_{\mathrm{NPSH}_{R}}=\frac{\mathrm{NPSH}_{R}}{u_{2}^{2} / g} .
$$

Referring to Figure $5, W_{1 b}=\left(V_{r_{1}} / \sin \beta_{b_{1}}\right)$, and thus

$$
C_{\mathrm{NPSH}_{R}}=\frac{\left(p_{\min }-p_{v}\right) / \gamma}{u_{2}^{2} / g}+\frac{1}{\sin ^{2} \beta_{b_{1}}} \frac{V_{r_{1}}^{2}}{2 u_{2}^{2}} .
$$

The term $\left(\left(p_{\min }-p_{v}\right) / \gamma\right) /\left(u_{2}^{2} / g\right)$ is a design parameter for the pump and could be assumed to be 0.02 .

From (3) $V_{r_{1}}=V_{r_{2}}\left(D_{2} / D_{1}\right)\left(b_{2} / b_{1}\right)\left(\varepsilon_{2} / \varepsilon_{1}\right)$, and therefore the $\mathrm{NPSH}_{R}$ coefficient is

$$
C_{\mathrm{NPSH}_{R}}=0.02+\frac{1}{2 \sin ^{2} \beta_{b_{1}}} \frac{(\psi / \varphi)^{2}}{\left(D_{1} / D_{2}\right)^{2}\left(b_{1} / b_{2}\right)^{2}\left(\varepsilon_{1} / \varepsilon_{2}\right)^{2}} .
$$

2.17. Pump Specific Speed $n_{s}$. The pump specific speed is defined with suitable units as

$$
n_{s}=\frac{N \sqrt{Q}}{H^{3 / 4}}[N(\mathrm{rpm}), H(\mathrm{~m}), \mathrm{Q}(\mathrm{lit} / \mathrm{s})] .
$$

Substitute for

$$
\begin{gathered}
Q=1000 \eta_{\mathrm{vol}} \varepsilon_{2} \cdot \pi D_{2} b_{2} \cdot \psi \sqrt{2 g H}(\text { lit } / \mathrm{s}), \\
N=\frac{60}{\pi D_{2}} \varphi \sqrt{2 g H} \text {, and } b_{2}=D_{2}\left(\frac{b_{2}}{D_{2}}\right) \text { yield : } \\
n_{s}=\frac{60 * \sqrt{1000} *(2 g)^{3 / 4} * \sqrt{\varepsilon_{2}}}{\sqrt{\pi}} \sqrt{\left(\frac{b_{2}}{D_{2}}\right)} * \sqrt{\eta_{\mathrm{vol}}} \cdot \varphi \sqrt{\psi}
\end{gathered}
$$

Therefore,

$$
n_{s}=9977 \sqrt{\varepsilon_{2}} \sqrt{\left(\frac{b_{2}}{D_{2}}\right)} \cdot \sqrt{\eta_{\mathrm{vol}}} \cdot \varphi \sqrt{\psi}
$$

\section{Calculation Procedure and Results}

An iterative procedure using Microsoft Office EXCEL program is performed to calculate the pump design parameters described by the preceding derived equations. The input parameters are the blades number, $Z$, impeller inlet diameter to outlet diameter ratio, $\left(D_{1} / D_{2}\right)$, volute diameter to impeller outlet diameter ratio, $\left(D_{3} / D_{2}\right)$, ratio of blade width at impeller outlet to impeller outlet diameter, $\left(b_{2} / D_{2}\right)$, blade width at impeller inlet to blade width at impeller outlet ratio, $\left(b_{1} / b_{2}\right)$, volute width to blade width at impeller outlet ratio, $\left(b_{3} / b_{2}\right)$, blade angle at impeller outlet, $\beta_{b_{2}}$, blade angle at 
TABle 1: Pump input-parameters.

\begin{tabular}{|c|c|c|}
\hline & Input parameter & Value \\
\hline \multirow{9}{*}{ Impeller } & $\frac{D_{1}}{D_{2}}$ & 0.5 \\
\hline & $\frac{b_{2}}{D_{2}}$ & 0.121 \\
\hline & $b_{1}^{2}$ & \multirow{2}{*}{1} \\
\hline & $b_{2}$ & \\
\hline & $\epsilon_{2} \quad y_{0}$ & 0.95 \\
\hline & $G=\frac{y_{0}}{\left(D_{2} / 2\right)}$ & \multirow[t]{2}{*}{0.05} \\
\hline & $\operatorname{Re}_{2}=\frac{u_{2} \cdot\left(D_{2} / 2\right)}{v}$ & \\
\hline & 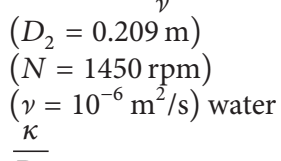 & $1.16 * 10^{6}$ \\
\hline & $\begin{array}{l}D_{2} \\
(\kappa=0.0001 \mathrm{~m})\end{array}$ & 0.000478 \\
\hline \multirow{5}{*}{ Blades } & $Z$ & 4 \\
\hline & $\beta_{b_{2}}$ & $164^{\circ}$ \\
\hline & $\beta_{b_{1}}$ & $164^{\circ}$ \\
\hline & $\frac{D_{3}}{D}$ & 1.05 \\
\hline & $\begin{array}{l}D_{2} \\
\frac{b_{3}}{b_{2}} \\
\end{array}$ & 1.05 \\
\hline \multirow{3}{*}{ Volute } & $\alpha_{V}$ & $7^{\circ}$ \\
\hline & $\alpha_{\mathrm{th}}$ & $4^{\circ}$ \\
\hline & $C_{d_{V}}$ & 0.8 \\
\hline \multirow{5}{*}{ Eye } & $D_{\text {eye }}$ & \multirow[t]{2}{*}{1} \\
\hline & $\overline{D_{y_{\text {th }}}}$ & \\
\hline & $\frac{y_{c}}{D_{\text {eye }}}$ & 0.005 \\
\hline & $C_{\mathrm{dL}}$ & 0.6 \\
\hline & $C_{\text {eye }}$ & 1.0 \\
\hline
\end{tabular}

impeller inlet, $\beta_{b_{1}}$, volute angle, $\alpha_{V}$, throat angle, $\alpha_{\mathrm{th}}$, ratio of wearing ring clearance/impeller eye diameter, $\left(y_{c} / D_{\text {eye }}\right)$, leakage discharge coefficient, $C_{\mathrm{dL}}$, volute diffusion loss coefficient, $C_{d_{V}}$, eye loss coefficient, $C_{\text {eye }}$, blade thickness coefficient at impeller outlet, $\varepsilon_{2}$, with constant-thickness blades, ratio of axial gap between impeller disk and casing to impeller outlet radius, $y_{0} /\left(D_{2} / 2\right)$, Reynolds number at impeller outlet, $\operatorname{Re}_{2}$, and relative roughness, $\left(\kappa / D_{2}\right)$ approximated values are used by putting $D_{2} \approx 0.209 \mathrm{~m}, N \approx 1450 \mathrm{rpm}, \kappa \approx 0.0001 \mathrm{~m}$, $\nu \approx 10^{-6} \mathrm{~m}^{2} / \mathrm{s}: \operatorname{Re}_{2}=1.16 * 10^{6}$, and $\kappa / D_{2}=0.000478$.

For a certain flow coefficient, $\psi$, all pump performance parameters and coefficients are calculated after iterations.

Case Study. For reason of comparison between results of present analytical study and experimental pump performance obtained by Baun and Flack [14], calculations of centrifugal pump performance are performed with the following input parameters given in Table 1.

Other pump constant-parameters are calculated according to the present procedure as shown in Table 2.

The sequence of calculations by using the procedure derived equations of pump variable parameters is listed in Table 3.

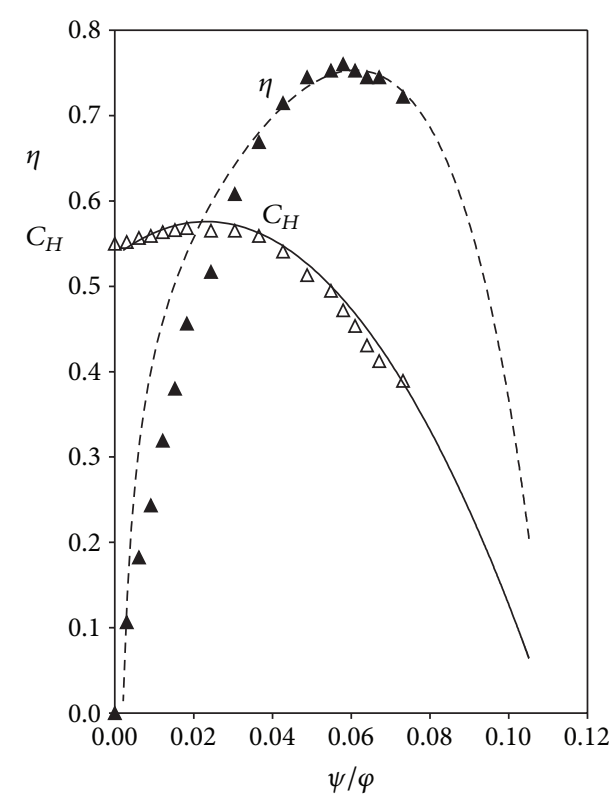

\footnotetext{
$\Delta \quad$ Experimental head coeff. [14]

— Present procedure head coeff. Efficiency

- Experimental [14]

- - - Present procedure
}

FIGURE 9: Comparison between present procedure results and experimental results by Baun and Flack [14].

The manometric head coefficient and pump efficiency of both present procedure and experimental measurements by Baun and Flack [14] are plotted in Figure 9 versus the ratio $(\psi / \varphi)$ (defined as flow coefficient in [14]). It shows a good similarity between the present procedure results and the experimental ones. Only in range of low ratios of $\psi / \varphi$, the calculated efficiency is relatively high. This is attributed to the uncounted mechanical power loss in the prediction of pump shaft power and hence in efficiency.

The pump performance characteristics are presented by the calculated pump head coefficient, efficiency, power coefficients, and required NPSH as shown in Figure 10. From the figure, the pump best efficiency point (BEP) occurs at discharge coefficient $C_{Q} \approx 0.0675$ giving an efficiency $\eta \approx$ $75.3 \%$, a manometric head coefficient $C_{H} \approx 0.468$, a shaft power coefficient $C_{P_{\mathrm{sh}}} \approx 0.414$, a water power coefficient $C_{P_{w}} \approx 0.312$, and a pump required net positive suction head coefficient $C_{\mathrm{NPSH}_{R}} \approx 0.129$.

The maximum shaft power coefficient $C_{P_{\mathrm{sh}}} \approx 0.428$ occurs at $C_{Q} \approx 0.085$, and the maximum water power coefficient $C_{P_{w}} \approx 0.3165$ occurs at $C_{\mathrm{Q}} \approx 0.075$.

The variations of pump flow coefficient $\psi$ and pump speed coefficient $\varphi$ with the discharge coefficient are shown in Figures 11 and 12, respectively. Figure 11 shows also the variation of the ratio $\psi / \varphi$ with the discharge coefficient $C_{Q}$. The linear relationship between $C_{Q}$ and $\psi / \varphi$ is evident as given by (6). The dotted lines in the figures correspond to the value of $C_{Q}$ that gives the pump best efficiency. At pump best efficiency point $\left(C_{Q} \approx 0.0675\right), \psi \approx 0.063$ (Figure 11) and $\varphi \approx 1.034$ (Figure 12). Also, it is indicated from Figure 12 that 
TABle 2: Pump constant-parameters.

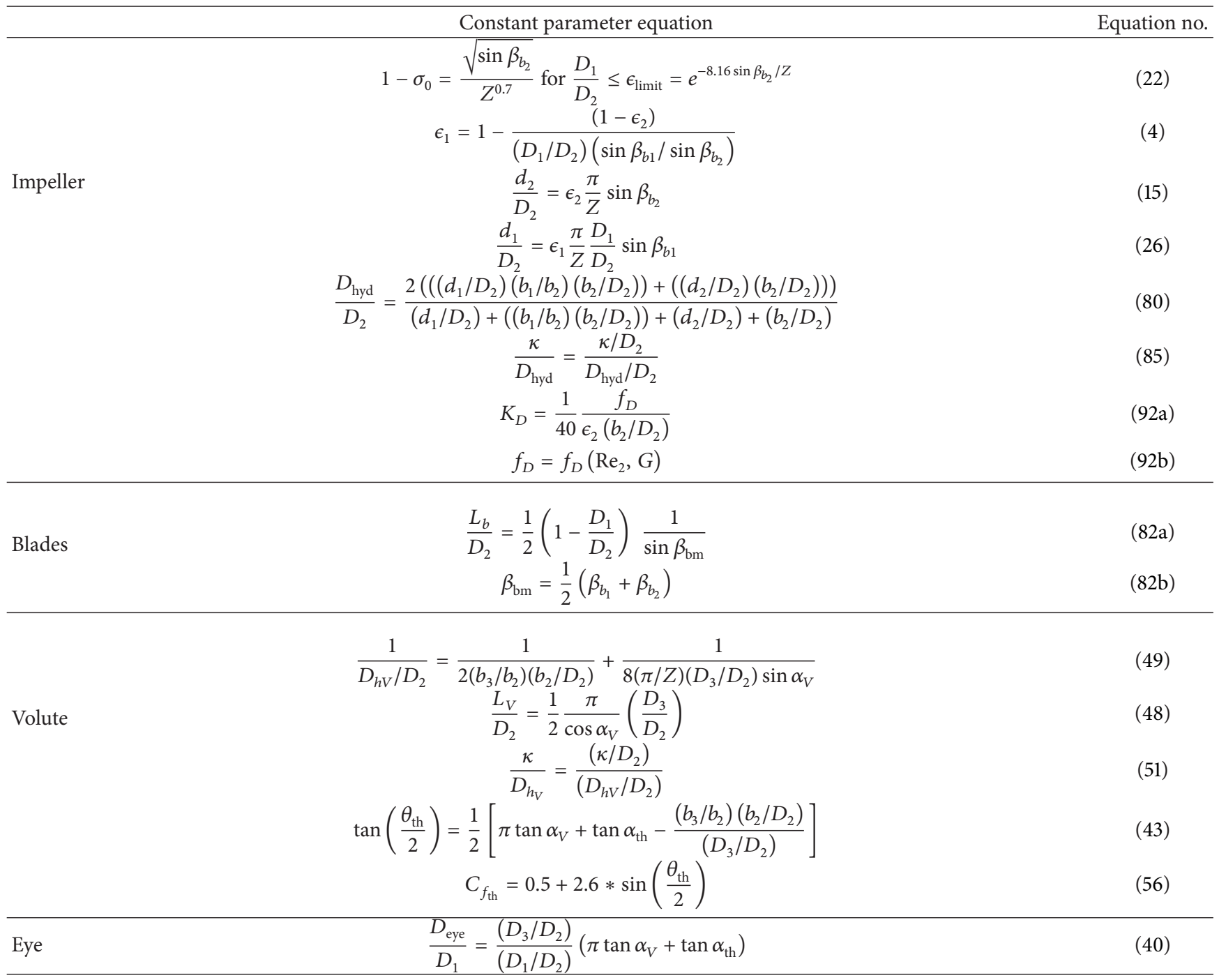

the speed coefficient takes a minimum value of $\varphi \approx 0.932$ at $C_{\mathrm{Q}} \approx 0.024$, which corresponds to the maximum $C_{H}$ in Figure 10 .

Figure 13 shows the theoretical dimensionless headdischarge curve (Euler head) which is a straight line, and $C_{H_{\infty}}$ decreases with the increase of $C_{Q}$ for the proposed outlet blade angle $\beta_{b_{2}}=164^{\circ}>90^{\circ}$. The actual impeller outlet dimensionless head-discharge curve $C_{H_{0}}$ is obtained taking into consideration the slip or eddy circulation of flow inside impeller which is nearly constant. Actually, the effect of slip is not a loss but a discrepancy not accounted by the basic assumptions. The second loss shown is the volute-and-eye loss, which is minimum at $C_{Q} \approx 0.051$. Reduced or increased $C_{\mathrm{Q}}$, from the value 0.051 , increases the volute-and-eye loss.

Figure 14 gives the variation of different head loss coefficients with $C_{Q}$ in addition to the $C_{H}$ and $C_{H_{0}}$ curves. Generally, these head loss coefficients decrease with the increase of $C_{Q}$. At very low discharge coefficients, both the disk head loss coefficient and volumetric head loss coefficient become very big. The inlet circulation head loss is big at low
$C_{Q}$ and decreases until it reaches zero at $C_{Q} \approx 0.06$, and then, its direction is inverse (becomes negative) which means that the inlet circulation adds power to impeller and does not bleed power from impeller.

The variation of the flow velocity coefficients and the pump volumetric efficiency $\eta_{\text {vol }}$ with the pump discharge coefficient is shown in Figure 15 . The coefficients $C_{V_{1}}, C_{V \Delta V_{u_{2}}}$, and $C_{V_{4}}$ have the trends of increasing with the increase of $C_{Q}$, whereas the two coefficients $C_{V_{3 d}}$ and $C_{V_{3 d^{\prime}}}$ have the trends of decreasing with the increase of $C_{Q}$. These two later coefficients have maximum values at $C_{Q}=0$. At $C_{Q} \approx 0.085$, the coefficient $C_{V_{3 d}}$ becomes zero, which indicates that at this point of operation the flow inside volute is without circulation and $V_{4}=V_{u_{2} \text { act }}$. At increased $C_{Q}$, the $C_{V_{3 d}}$ becomes negative which means that the flow circulation inside the volute changes its direction of rotation opposite to impeller motion whereas $V_{4}>V_{u_{2} \text { act }}$ (Figure 6).

The pump specific speed is presented in Figure 16, which shows that $n_{s} \approx 870$ at pump best efficiency point. 
TABle 3: Pump variable-parameters.

Order

\begin{tabular}{|c|c|}
\hline & $\psi \equiv \frac{V_{r_{2}}}{\sqrt{2 g H}}=0.002$ to $0.12 \quad$ step $=0.001$ \\
\hline Initial & $\sigma=\sigma_{0}, x=1, \eta_{\mathrm{vol}}=1$ \\
\hline & Start of iteration \\
\hline 1 & $\varphi=-\frac{1}{2} \psi \cot \beta_{b_{2}}+\frac{1}{2} \sqrt{\psi^{2} \cot ^{2} \beta_{b_{2}}+\frac{2}{x \sigma}}$ \\
\hline 2 & $y=C_{H_{\infty}} \equiv \frac{H_{\infty}}{u_{2}^{2} / g}=1+\frac{\psi}{\varphi} \cot \beta_{b_{2}}$ \\
\hline 3 & $\sigma=1-\frac{\left(1-\sigma_{0}\right)}{y}$ \\
\hline 4 & $C_{h_{l_{\text {slp }}}} \equiv \frac{h_{l_{\text {slp }}}}{u_{2}^{2} / g}=(1-\sigma) C_{H_{\infty}}$ \\
\hline 5 & $C_{H_{0}} \equiv \frac{H_{0}}{u_{2}^{2} / g}=\sigma\left(1+\frac{\psi}{\varphi} \cot \beta_{b 2}\right)$ \\
\hline
\end{tabular}

6

$$
C_{V_{5}} \equiv \frac{V_{5}}{\sqrt{2 g H}}=\frac{4 \epsilon_{2} \eta_{\mathrm{vol}}\left(b_{2} / D_{2}\right)}{\left(D_{t h} / D_{3}\right)^{2}\left(D_{3} / D_{2}\right)^{2}} \cdot \psi
$$

7

$$
C_{V_{4}} \equiv \frac{V_{4}}{\sqrt{2 g H}}=\frac{\epsilon_{2} \eta_{\mathrm{vol}}}{\left(D_{3} / D_{2}\right)\left(b_{3} / b_{2}\right) \tan \alpha_{V}} \cdot \psi
$$

8

$$
C_{V_{\text {eye }}} \equiv \frac{V_{\text {eye }}}{\sqrt{2 g H}}=\frac{4 \epsilon_{2}\left(b_{2} / D_{2}\right)}{\left(D_{t h} / D_{3}\right)^{2}\left(D_{3} / D_{2}\right)^{2}} \cdot \psi
$$

9

$$
C_{V_{3 p}}=\frac{C_{V 4}}{\cos \alpha_{V}}
$$

10

$$
C_{V_{3 d}}=\sigma\left(\varphi+\psi \cot \beta_{b 2}\right)-C_{V 4}
$$

11

$$
C_{V_{3 r}} \equiv \frac{V_{3 r}}{\sqrt{2 g H}}=\frac{\epsilon_{2} \eta_{\mathrm{vol}}}{\left(D_{3} / D_{2}\right)\left(b_{3} / b_{2}\right)} \cdot \psi
$$

$12 \quad \eta_{\mathrm{vol}}=1-\left(\frac{y_{c}}{D_{\text {eye }}}\right)\left(\frac{D_{\text {eye }}}{D_{1}}\right)^{2}\left(\frac{D_{1}}{D_{2}}\right)^{2} \frac{1}{\left(b_{2} / D_{2}\right)} \frac{\sqrt{2} C_{\mathrm{dL}}}{\epsilon_{2}} \frac{\varphi}{\psi} * \sqrt{C_{H_{0}}-\frac{C_{V_{4}}^{2}-C_{V_{5}}^{2}+C_{\text {eye }} \cdot C_{V_{\text {eye }}}^{2}}{2 \varphi^{2}}-\frac{1}{8}\left[1-\left(\frac{D_{1}}{D_{2}}\right)^{2}\left(\frac{D_{\text {eye }}}{D_{1}}\right)^{2}\right]}$ 
TABle 3: Continued.

14

$f_{V}=\frac{0.3086}{\left\{\log \left[\left(6.9 / \operatorname{Re}_{V}\right)+\left(\left(\kappa / D_{h V}\right) / 3.7\right)^{1.11}\right]\right\}^{2}}$

15

$$
C_{f_{V}}=f_{V} \frac{L_{V}}{D_{h V}}=f_{V}\left(\frac{L_{V}}{D_{2}}\right)\left(\frac{1}{D_{h V} / D_{2}}\right)
$$

16

$$
\frac{h_{l_{V}}}{H}=C_{f_{V}} C_{V_{3 p}}^{2}+C_{d_{V}} C_{V_{3 d}}^{2}+C_{V_{3 r}}^{2}+C_{f_{t h}} C_{V_{4}}^{2}
$$

17

$$
\frac{h_{l_{\text {eye }}}}{H}=C_{\text {eye }} \cdot C_{V_{\text {eye }}}^{2}
$$

18

$$
\frac{1}{x}=1+\frac{h_{l_{V}}}{H}+\frac{h_{l_{\text {eye }}}}{H}
$$

\section{End of iteration}

19

$$
C_{h_{l_{V+\text { eye }}}} \equiv \frac{h_{l_{V}}+h_{l_{\text {eye }}}}{u_{2}^{2} / g}=(1-x) \sigma C_{H_{\infty}}
$$

20

$$
C_{H} \equiv \frac{H}{u_{2}^{2} / g}=x \sigma\left(1+\frac{\psi}{\varphi} \cot \beta_{b_{2}}\right)
$$

21

$$
C_{\mathrm{Q}} \equiv \frac{\mathrm{Q}}{(N / 60) D_{2}^{3}}=\eta_{\mathrm{vol}} \epsilon_{2} \pi^{2}\left(\frac{b_{2}}{D_{2}}\right) \cdot\left(\frac{\psi}{\varphi}\right)
$$

22

$$
\frac{W_{\mathrm{av}}}{u_{2}}=\frac{\epsilon_{2}(\pi / Z)\left(b_{2} / D_{2}\right) \cdot(\psi / \varphi)}{1 / 2\left(\left(\left(d_{1} / D_{2}\right)\left(b_{1} / b_{2}\right)\left(b_{2} / D_{2}\right)\right)+\left(\left(d_{2} / D_{2}\right)\left(b_{2} / D_{2}\right)\right)\right)}
$$

$$
\operatorname{Re}=\frac{W_{\text {av }} \cdot D_{\text {hyd }}}{v}=2\left(\frac{W_{\text {av }}}{u_{2}}\right)\left(\frac{D_{\text {hyd }}}{D_{2}}\right) \cdot \operatorname{Re}_{2}
$$

$$
f_{i}=\frac{0.3086}{\left\{\log \left[(6.9 / \mathrm{Re})+\left(\left(\kappa / D_{\text {hyd }}\right) / 3.7\right)^{1.11}\right]\right\}^{2}}
$$


TABle 3: Continued.

$$
C_{h_{l D}} \equiv \frac{h_{l_{D}}}{u_{2}^{2} / g}=K_{D} \cdot \frac{1}{\eta_{\mathrm{vol}}} \frac{\varphi}{\psi}
$$

$$
C_{h_{l_{\mathrm{cir} \text { in }}}} \equiv \frac{h_{l_{\mathrm{cir}_{\text {in }}}}}{u_{2}^{2} / g}=\left(1-\epsilon_{1} \frac{\pi}{Z} \sin \beta_{b 1}\right)\left(\frac{D_{1}}{D_{2}}\right)^{2}-\frac{\cot \left(180-\beta_{b 1}\right)}{\left(b_{1} / b_{2}\right)\left(\epsilon_{1} / \epsilon_{2}\right)} \cdot \frac{\psi}{\varphi}
$$

$$
C_{h_{l \mathrm{vol}}} \equiv \frac{h_{l_{\mathrm{vol}}}}{u_{2}^{2} / g}=\left(\frac{1}{\eta_{\mathrm{vol}}}-1\right) \cdot\left(C_{H_{0}}+C_{h_{l f}}+C_{h_{l \mathrm{cir} \text { in }}}\right)
$$

30

$$
C_{H_{\mathrm{sh}}} \equiv \frac{H_{\mathrm{sh}}}{u_{2}^{2} / g}=C_{H_{0}}+C_{h_{l f}}+C_{h_{l \mathrm{cir}}}+C_{h_{l \mathrm{vol}}}+C_{h_{l D}}
$$

31

$$
\eta \equiv \frac{P_{w}}{P_{\mathrm{sh}}}=\frac{C_{H}}{C_{H_{\mathrm{sh}}}}
$$

$$
C_{P_{\mathrm{sh}}} \equiv \frac{P_{\mathrm{sh}}}{\rho(N / 60)^{3} D_{2}^{5}}=\pi^{2} C_{\mathrm{Q}} C_{H_{\mathrm{sh}}}
$$

$$
C_{P_{w}} \equiv \frac{P_{w}}{\rho(N / 60)^{3} D_{2}^{5}}=\pi^{2} C_{Q} C_{H}
$$

$$
C_{\mathrm{NPSH}_{R}}=0.02+\frac{1}{2 \sin ^{2} \beta_{b_{1}}} \frac{(\psi / \varphi)^{2}}{\left(D_{1} / D_{2}\right)^{2}\left(b_{1} / b_{2}\right)^{2}\left(\epsilon_{1} / \epsilon_{2}\right)^{2}}
$$

$$
n_{s}=9977 \sqrt{\epsilon_{2}} \sqrt{\left(\frac{b_{2}}{D_{2}}\right)} \cdot \sqrt{\eta_{\mathrm{vol}}} \cdot \varphi \sqrt{\psi}
$$

Figure 17 shows the procedure results of centrifugal pump performance when the pump handles fluids with different kinematic viscosities. The head and efficiency for the pump when handling oils are lower than those when handling water. But the required power when handling oil is higher than that when handling water.

The pump head decreases slightly due to the increase in volute friction loss as fluid viscosity increases, while the increase in pump power is high due to the increase in both hydraulic friction inside impeller and disk friction power losses. Hence, the drop in pump efficiency is very high as the fluid viscosity increases. This result is in accordance with experimental results by Shojaee Fard and Boyaghchi [15].

\section{Conclusions}

A one-dimensional flow procedure for analytical study of centrifugal pump performance is accomplished applying the principle theories of turbomachines. The procedure is capable of providing the performance characteristic of centrifugal pump in a dimensionless information form. The predicted coefficients and performance curves obtained have been found to be in a reasonable agreement with experimental measurements. The present procedure is also capable of predicting the effects of handling viscous fluids on the centrifugal pump performance. The input form for this procedure of pump flow analysis makes it an effective tool analysis and can be used in the pump conceptual design.

\section{Notations}

$A$ : Impeller net area, $\mathrm{m}^{2}$

$a_{c}$ : Clearance area of wearing ring, $\mathrm{m}^{2}$

$A_{V}$ : Volute area, $\mathrm{m}^{2}$

$A_{\text {th }}$ : Throat outlet area, $\pi D_{\text {th }}^{2} / 4, \mathrm{~m}^{2}$

$A_{V_{\mathrm{th}}}$ : Volute throat inlet area, $\mathrm{m}^{2}$ 


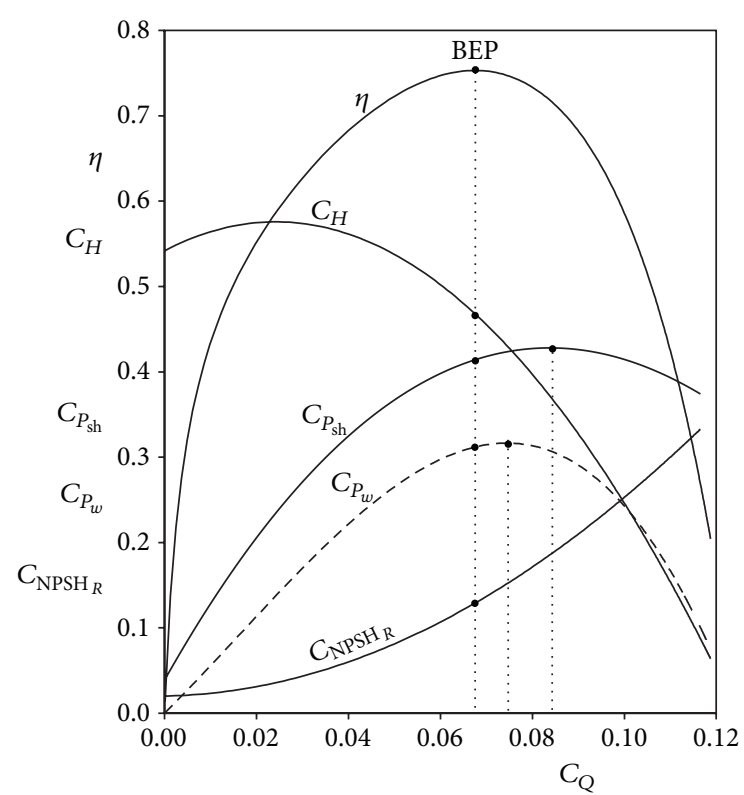

FIGURE 10: Characteristics of centrifugal pump obtained by present study.

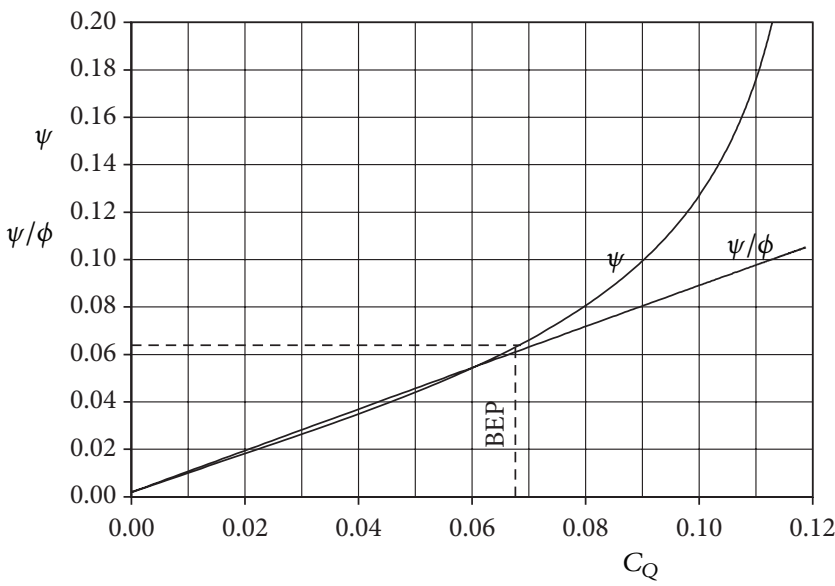

FIGURE 11: Variation of flow coefficient and ratio of flow coefficient to speed coefficient with discharge coefficient.

$b: \quad$ Impeller width, $\mathrm{m}$

$C_{d_{i}}$ : Impeller dissipation coefficient

$C_{\mathrm{dL}}$ : Leakage discharge coefficient

$C_{d_{V}}$ : Volute diffusion loss coefficient

$C_{\text {eye }}$ : Eye loss coefficient

$C_{f_{\text {th }}}$ : Volute throat friction loss coefficient

$C_{f_{\mathrm{V}}}$ : Volute friction loss coefficient

$C_{H}$ : Manometric head coefficient, $H /\left(u_{2}^{2} / g\right)$

$C_{H_{0}}$ : Head coefficient at impeller outlet, $H_{0} /\left(u_{2}^{2} / g\right)$

$C_{H_{\infty}}$ : Euler head coefficient, $H_{\infty} /\left(u_{2}^{2} / g\right)$

$C_{H_{\mathrm{sh}}}$ : Shaft head coefficient, $H_{\mathrm{sh}} /\left(u_{2}^{2} / g\right)$

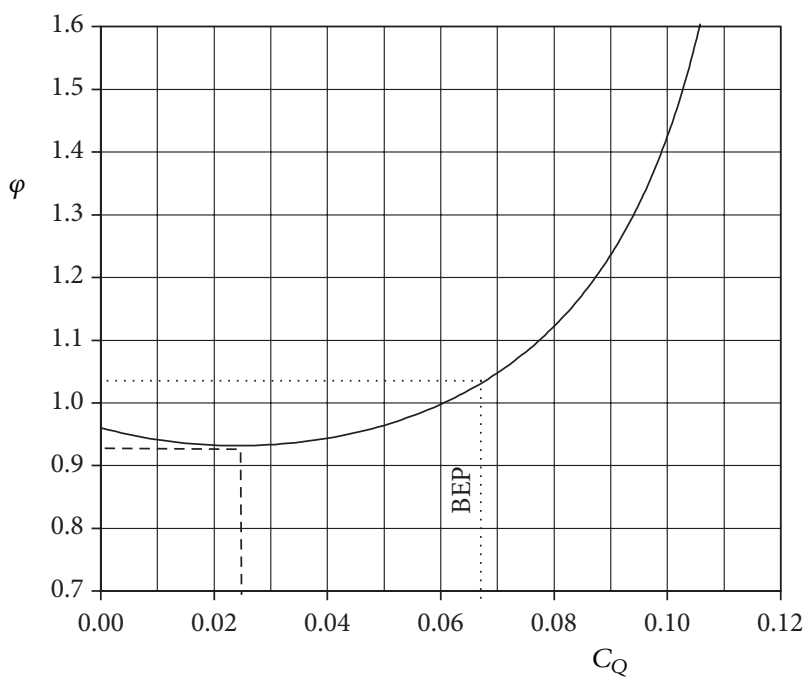

FIGURE 12: Speed coefficient variation with pump discharge coefficient.

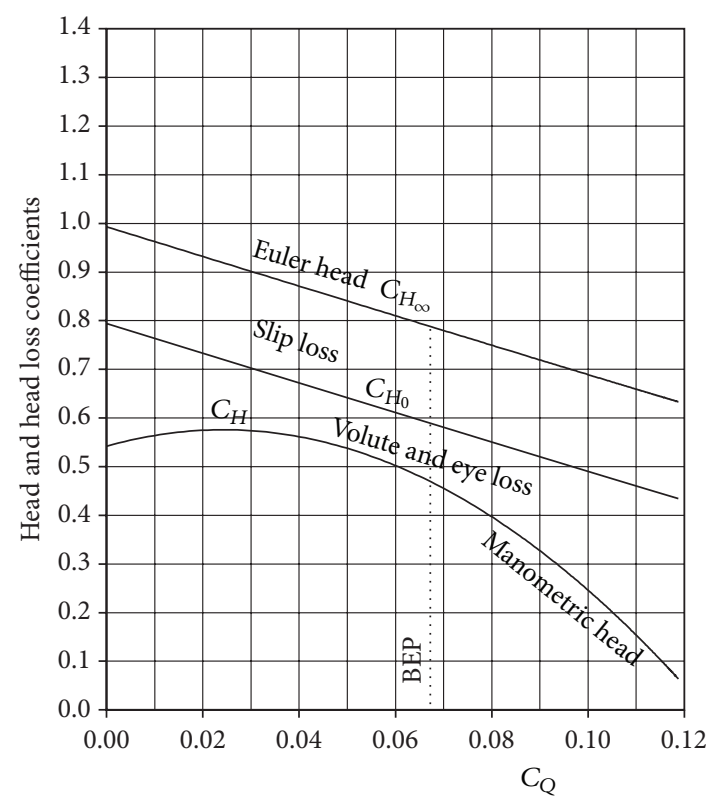

FIGURE 13: Variation of head coefficients and head loss coefficients with discharge coefficient.

$C_{h_{l_{\text {cir }}}}$ : Inlet shock circulation head loss coefficient, $h_{l_{\text {cirin }}} /\left(u_{2}^{2} / g\right)$

$C_{h_{l f}}:$ Impeller skin friction head loss coefficient

$C_{h_{l_{\text {eye }}}}$ : Eye head loss coefficient, $h_{l_{\text {eye }}} /\left(u_{2}^{2} / g\right)$

$C_{h_{l_{D}}}$ : Disk friction head loss coefficient, $h_{l_{D}} /\left(u_{2}^{2} / g\right)$

$C_{h_{l s p}}: \quad$ Slip head loss coefficient, $h_{l_{\text {spp }}} /\left(u_{2}^{2} / g\right)$

$C_{h_{l_{V}}}: \quad$ Volute head loss coefficient, $h_{l_{V}} /\left(u_{2}^{2} / g\right)$

$C_{h_{l_{V+e y e}}}$ : Volute-eye head loss coefficient, $\left(h_{l_{V}}+h_{l_{\text {eye }}}\right) /\left(u_{2}^{2} / g\right)$

$C_{h_{l_{\mathrm{vol}}}}$ : Volumetric head loss coefficient, $h_{l_{\mathrm{vol}}} /\left(u_{2}^{2} / g\right)$ 


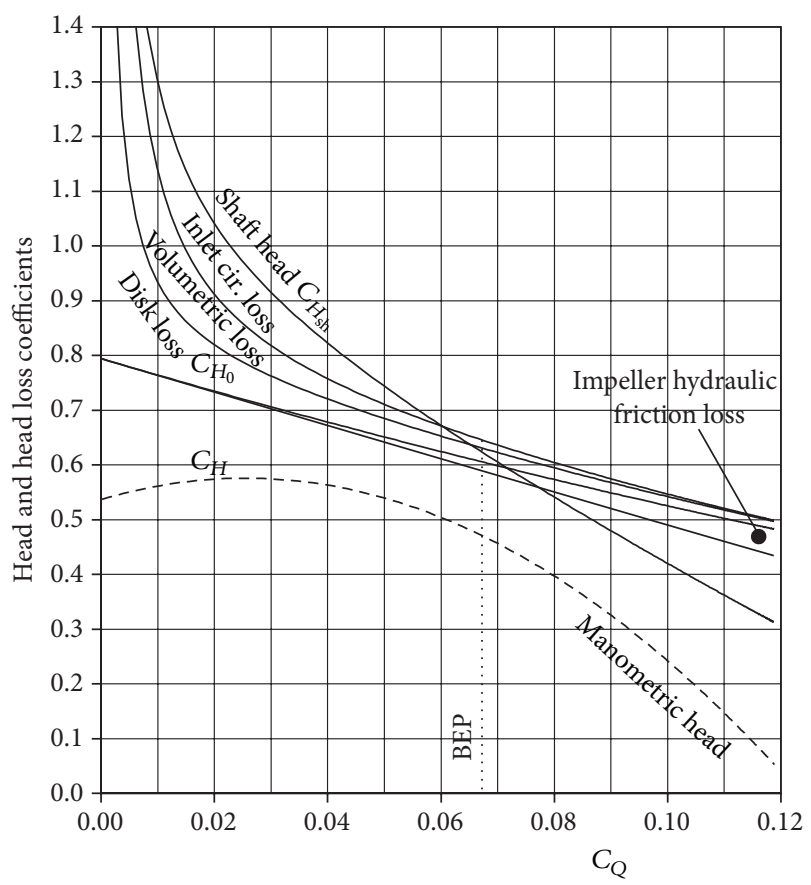

Figure 14: Head loss coefficients affecting pump power at different discharge coefficients.

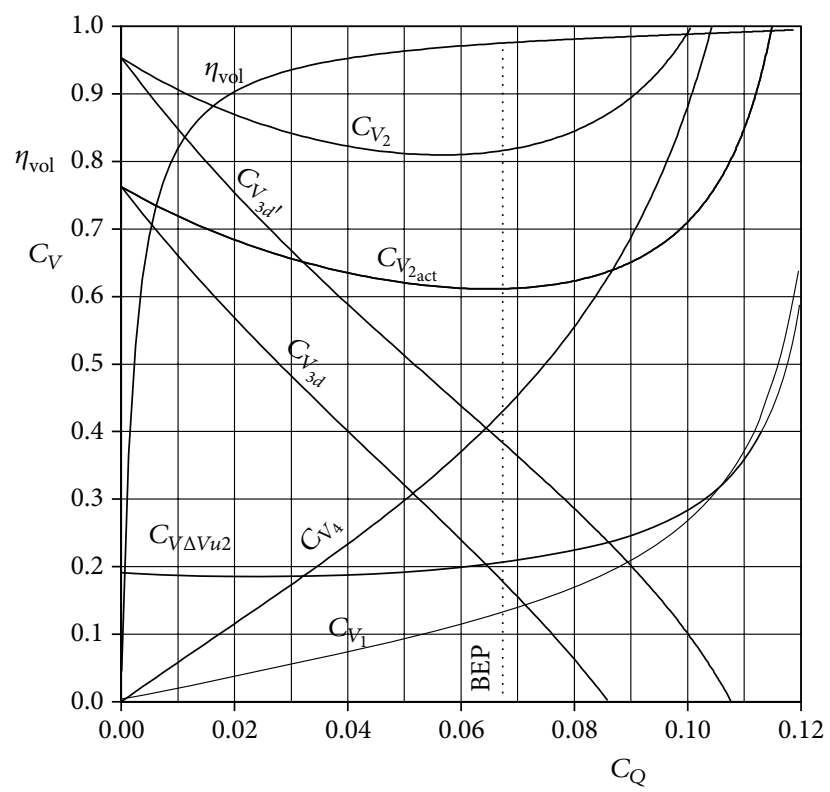

Figure 15: Values of volumetric efficiency and different pump velocity coefficients.
$C_{\mathrm{NPSH}_{R}}$ : Coefficient of pump NPSH${ }_{R}, \mathrm{NPSH}_{R} /\left(u_{2}^{2} / g\right)$
$C_{P_{\mathrm{sh}}}: \quad$ Shaft power coefficient, $P_{\mathrm{sh}} / \rho(N / 60)^{3} D_{2}^{5}$
$C_{P_{w}}: \quad$ Water power coefficient, $P_{w} / \rho(N / 60)^{3} D_{2}^{5}$
$C_{\mathrm{Q}}$ : $\quad$ Pump discharge coefficient, $\mathrm{Q} /(\mathrm{N} / 60) D_{2}^{3}$
$C_{\mathrm{Q}_{L}}$ : $\quad$ Leakage flow rate coefficient
$C_{V}: \quad$ Velocity coefficient, $V / \sqrt{2 g H}$
$D_{2}$ : Impeller outer diameter, $\mathrm{m}$

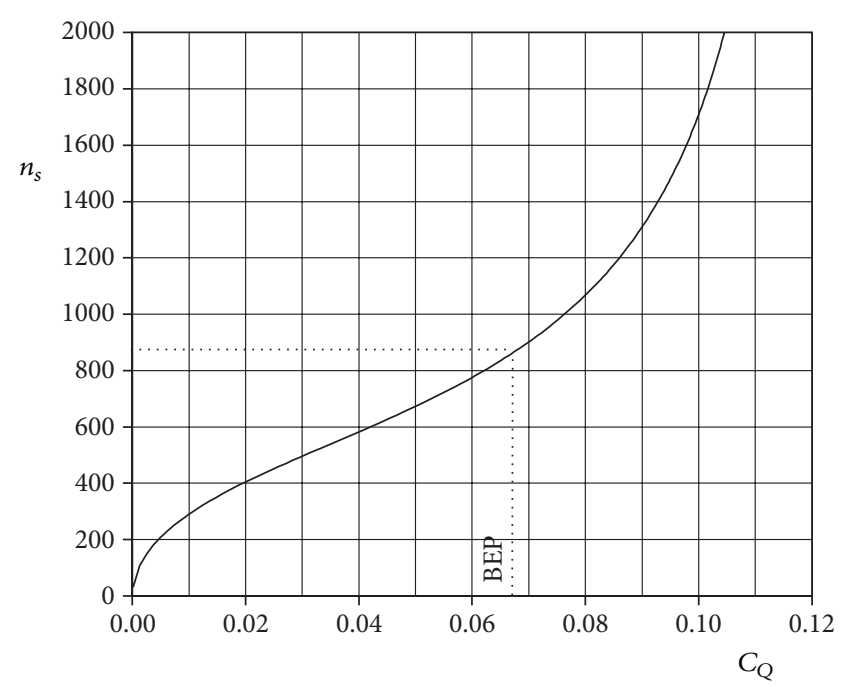

Figure 16: Pump specific speed values at different discharge coefficient.

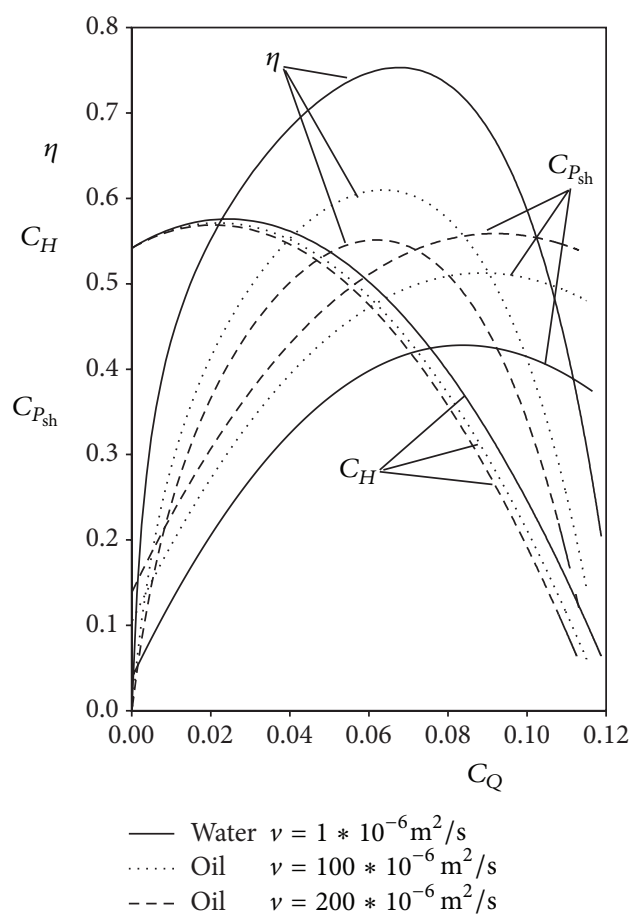

FIGURE 17: Effect of pumped fluid viscosity on the performance of centrifugal pump.

$D_{\text {eye }}:$ Pump eye diameter, $m$

$D_{h_{V}}:$ Average volute hydraulic diameter, $\mathrm{m}$

$D_{\text {th }}$ : Throat diameter, $\mathrm{m}$

$f: \quad$ Hydraulic friction coefficient

$f_{D}$ : Disk friction coefficient

$g$ : Acceleration of gravity, $\mathrm{m} / \mathrm{s}^{2}$

$G$ : Impeller disk gap parameter, $y_{0} /\left(D_{2} / 2\right)$

$h_{l_{\text {cirin }}}:$ Inlet shock circulation head loss, $\mathrm{m}$

$h_{l_{D}}:$ Disk friction head loss, $\mathrm{m}$ 
$h_{l_{f}}: \quad$ Impeller skin friction head loss, $\mathrm{m}$

$h_{l_{\mathrm{sp}}}: \quad$ Slippage head loss, $\mathrm{m}$

$h_{l_{V}}: \quad$ Volute head loss, $\mathrm{m}$

$h_{l_{\mathrm{vol}}}: \quad$ Volumetric head loss, $\mathrm{m}$

$H$ : $\quad$ Manometric head of pump, $m$

$H_{0}$ : Water head at impeller outlet, $\mathrm{m}$

$H_{\text {sh }}$ : Pump shaft head, m

$H_{\infty}$ : $\quad$ Euler pump head, $\mathrm{m}$

$K_{D}$ : Impeller disk loss coefficient

$L_{b}: \quad$ Blade length, $\mathrm{m}$

$L_{\text {th }}: \quad$ Throat length, $\mathrm{m}$

$L_{V}$ : $\quad$ Volute length, $\mathrm{m}$

$N$ : $\quad$ Pump rotational speed, rpm

NPSH: Pump net positive suction head, $\mathrm{m}$

$\mathrm{NPSH}_{R}$ : Pump required net positive suction head, $\mathrm{m}$

$n_{s}: \quad$ Pump specific speed

p: $\quad$ Pressure, $\mathrm{N} / \mathrm{m}^{2}$

$p_{c}: \quad$ Pressure before wearing ring clearance, $\mathrm{N} / \mathrm{m}^{2}$

$p_{s}: \quad$ Pressure at pump suction side, $\mathrm{N} / \mathrm{m}^{2}$

$P_{\text {sh }}: \quad$ Pump shaft power, W

$P_{w}: \quad$ Pumped water power, $\mathrm{W}$

$P_{\mathrm{sh}_{0}}: \quad$ Impeller Euler power, $\mathrm{W}$

$P_{l_{\text {cirin }}}$ : Inlet shock circulation power loss, $\mathrm{W}$

$P_{l_{f}}: \quad$ Impeller friction power loss, $\mathrm{W}$

$P_{l_{D}}: \quad$ Disk friction power loss, $\mathrm{W}$

$P_{l_{\text {vol }}}: \quad$ Volumetric power loss, $\mathrm{W}$

$Q: \quad$ Pump discharge, $\mathrm{m}^{3} / \mathrm{s}$

$Q_{i}: \quad$ Impeller discharge, $\mathrm{m}^{3} / \mathrm{s}$

$Q_{L}: \quad$ Pump internal discharge leakage, $\mathrm{m}^{3} / \mathrm{s}$

$r: \quad$ Radius, $m$

$t$ : $\quad$ Blade thickness, $m$

$u$ : Tangential flow velocity, $\mathrm{m} / \mathrm{s}$

$V: \quad$ Absolute flow velocity, $\mathrm{m} / \mathrm{s}$

$V_{3 p}$ : Throughflow component of volute velocity, $\mathrm{m} / \mathrm{s}$

$V_{3 d}: \quad$ Circulation velocity of flow in volute, $\mathrm{m} / \mathrm{s}$

$W: \quad$ Relative velocity of flow, $\mathrm{m} / \mathrm{s}$

$x: \quad$ Ratio, $H / H_{0}$

$y: \quad$ Group parameter, $\left(y=C_{H_{\infty}}\right)$

$y_{0}$ : Axial gap between impeller disk and casing, $\mathrm{m}$

$y_{c}: \quad$ Clearance of wearing ring, $\mathrm{m}$

$Z$ : $\quad$ Number of blades.

\section{Greek Letters}

$\alpha$ : Theoretical absolute velocity angle, degrees

$\alpha_{V}:$ Volute angle, degrees

$\beta_{b}$ : Blade angle, degrees

$\theta_{\text {th }}$ : Volute throat angle, degrees

$\epsilon$ : Blade thickness coefficient

$\varphi$ : Pump speed coefficient, $u_{2} / \sqrt{2 g H}$

$\eta$ : $\quad$ Manometric efficiency of pump

$\eta_{\mathrm{vol}}$ : Volumetric efficiency

$v$ : Fluid kinematic viscosity, $\mathrm{m}^{2} / \mathrm{s}$

$\rho: \quad$ Density of fluid, $\mathrm{kg} / \mathrm{m}^{3}$

$\sigma: \quad$ Slip factor, $V_{u_{2 \text { act }}} / V_{u_{2}}$ $\psi$ : Pump flow coefficient, $V_{r_{2}} / \sqrt{2 g H}$

$\kappa$ : Roughness height, $\mathrm{m}$.

\section{Subscripts}

1: Impeller inlet

2: Impeller outlet

3: Volute inlet

4: Volute outlet (throat inlet)

5: Pump outlet (throat outlet)

av: Average

act: Actual

eye: Pump eye

c: Clearance

hl: Head loss

$i$ : Impeller

l: $\quad$ Loss

$r$ : Radial direction

$s$ : Suction

sh: Shaft

th: Volute throat

$u$ : Tangential direction

vol: Volumetric.

\section{References}

[1] M. Asuaje, F. Bakir, S. Kouidri, F. Kenyery, and R. Rey, "Numerical modelization of the flow in centrifugal pump: volute influence in velocity and pressure fields," International Journal of Rotating Machinery, vol. 2005, no. 3, pp. 244-255, 2005.

[2] H. W. Oh and M. K. Chung, "Optimum values of design variables versus specific speed for centrifugal pumps," Proceedings of the Institution of Mechanical Engineers A, vol. 213, no. 3, pp. 219-226, 1999.

[3] H. W. Oh and M. K. Chung, "Design and performance analysis of centrifugal pump," World Academy of Science, Engineering and Technology, vol. 36, pp. 422-429, 2008.

[4] R. A. van den Braembussche, "Flow and loss mechanisms in volutes of centrifugal pumps," in Design and Analysis of High Speed Pumps, pp. 121-12-26, Educational Notes RTO-EN-AVT143, Paper 12, Neuilly-sur-Seine, France, 2006.

[5] J. Tuzson, Centrifugal Pump Design, John Wiley \& Sons, New York, NY, USA, 2000.

[6] E. Logan Jr. and R. Roy, Handbook of Turbomachinery, Marcel Dekker, New York, NY, USA, 2003.

[7] S. L. Dixon, Fluid Mechanics and Thermodynamics of Turbomachinery, Elsevier Butterworth-Heinemann, New York, NY, USA, 5th edition, 2005.

[8] F. J. Wiesner, "A review of slip factors for centrifugal impellers," Journal for Engineering for Power, vol. 89, no. 4, pp. 558-572, 1967.

[9] C. E. Brennen, Hydrodynamics of Pump, Oxford University Press, Oxford, UK, 1994.

[10] B. K. Hodge, Analysis and Design of Energy Systems, PrenticeHall, New York, NY, USA, 2nd edition, 1990.

[11] J. F. Gülich, Centrifugal Pumps, Springer, Berlin, Germany, 2010.

[12] N. P. Kruyt, Lecture Notes: Fluid Mechanics of Turbomachines II, Turbomachinery Laboratory, University of Twente, Amsterdam, The Netherlands, 2009. 
[13] K. M. Srinivasan, Rotodynamic Pumps (Centrifugal and Axial), New Age International, New Delhi, India, 2008.

[14] D. O. Baun and R. D. Flack, "Effects of volute design and number of impeller blades on lateral impeller forces and hydraulic performance," International Journal of Rotating Machinery, vol. 9, no. 2, pp. 145-152, 2003.

[15] M. H. Shojaee Fard and F. A. Boyaghchi, "Studies on the influence of various blade outlet angles in a centrifugal pump when handling viscous fluids," American Journal of Applied Sciences, vol. 4, no. 9, pp. 718-724, 2007. 

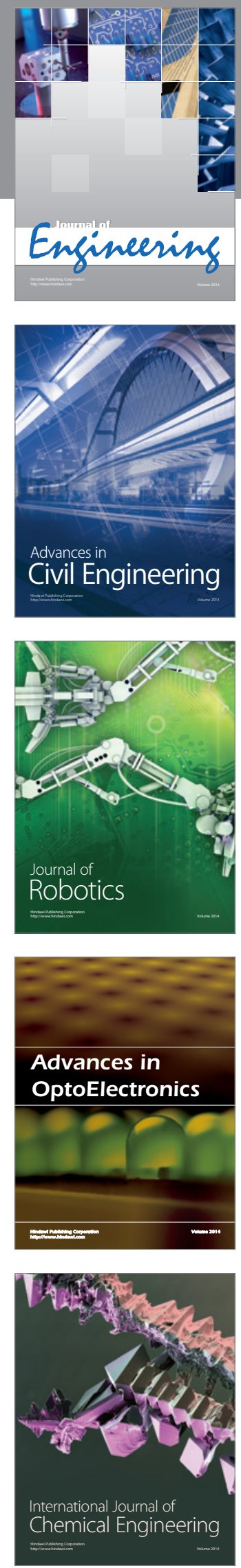

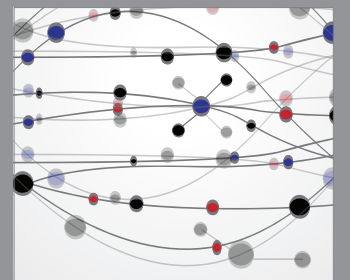

The Scientific World Journal
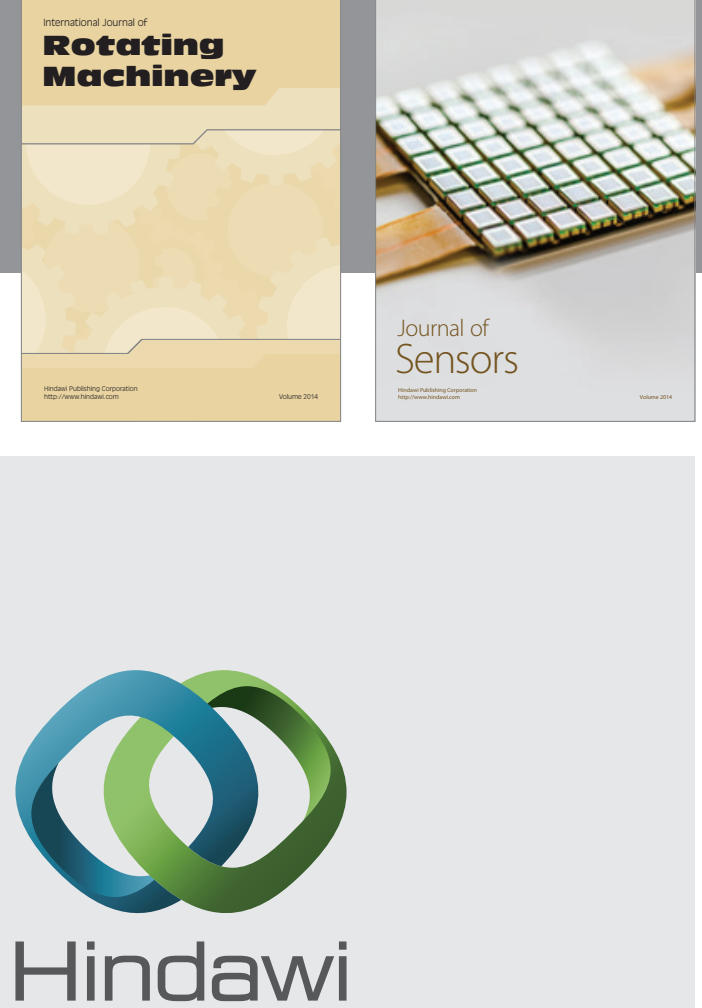

Submit your manuscripts at http://www.hindawi.com
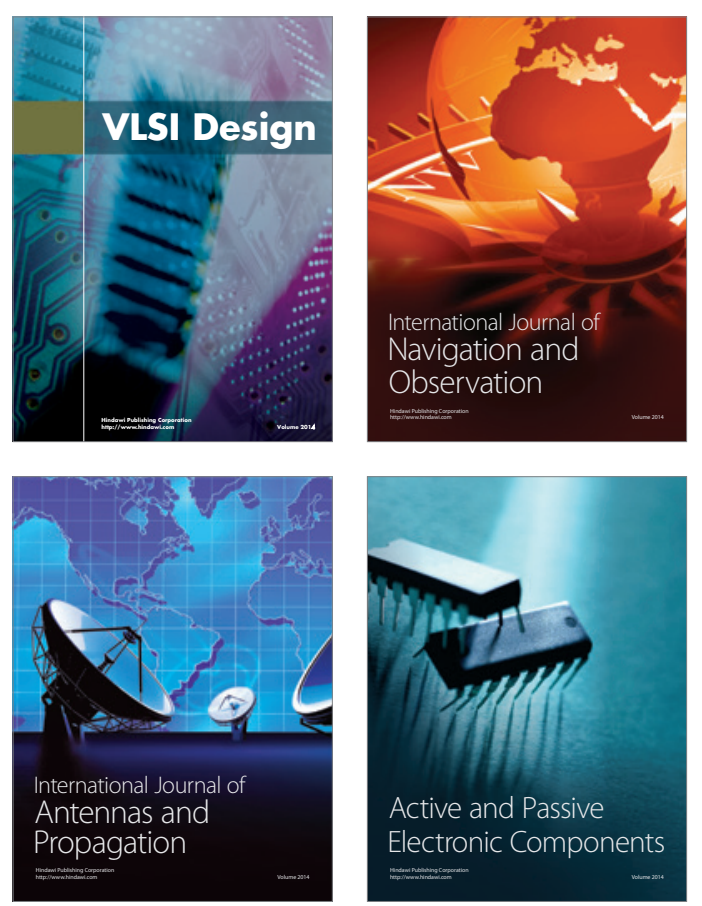
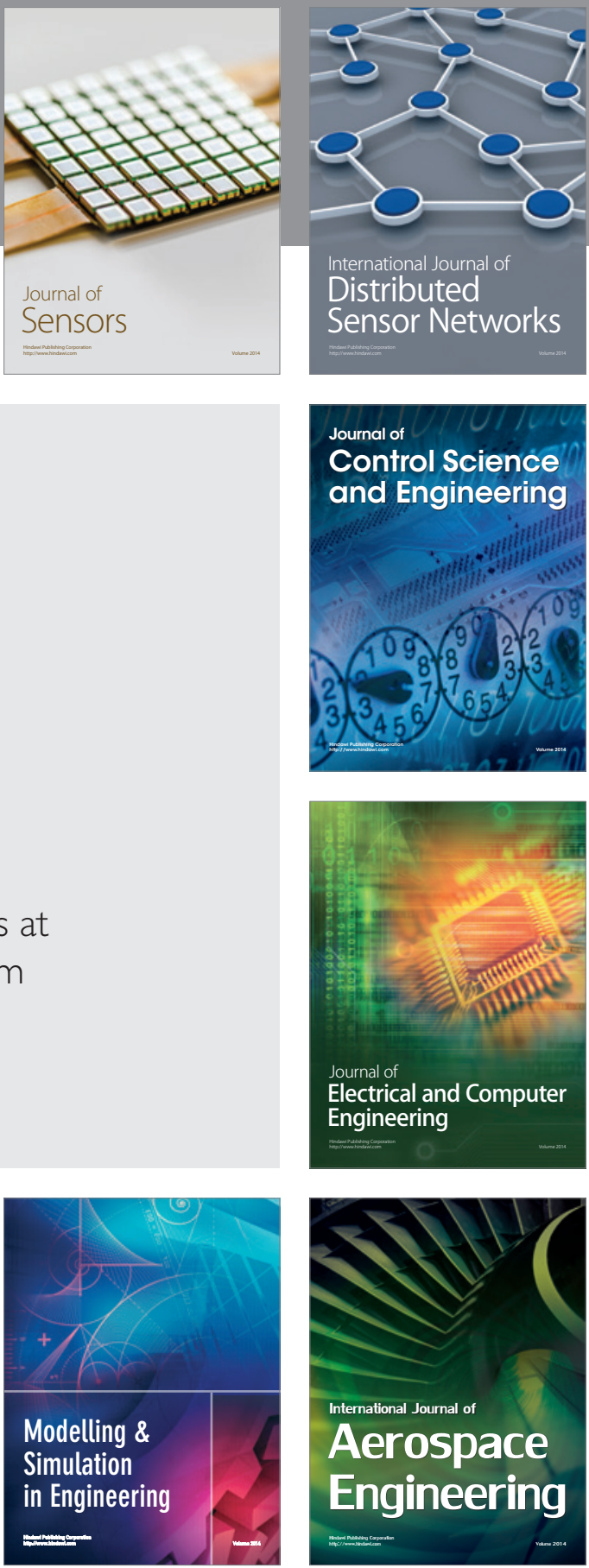

Journal of

Control Science

and Engineering
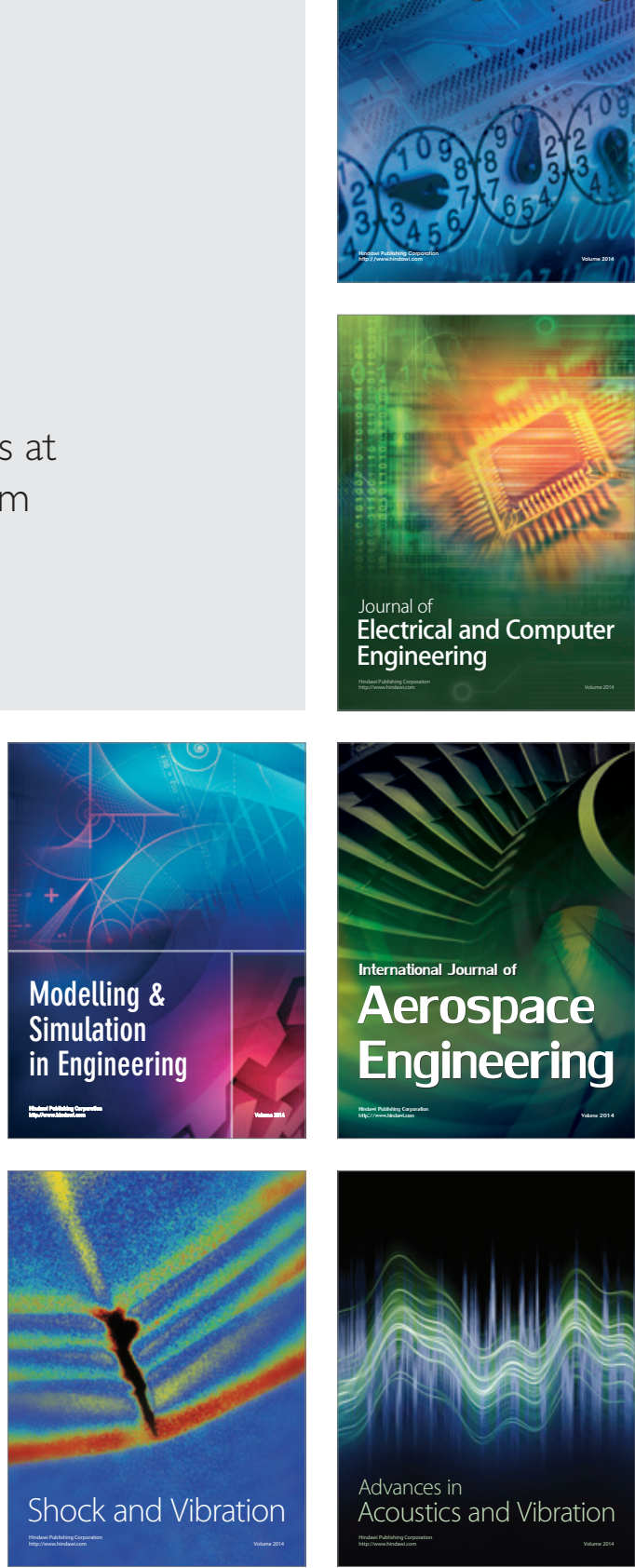